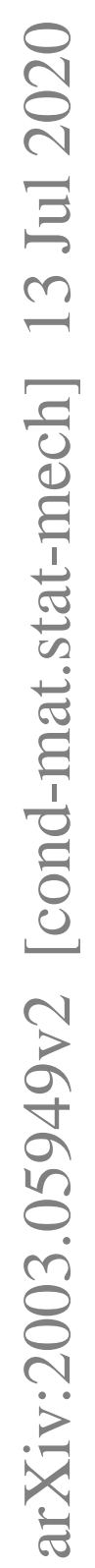




\title{
Collisionless kinetic theory for parametrically pumped magnons
}

Viktor Hahn ${ }^{\mathrm{a}}$ and Peter Kopietz

Institut für Theoretische Physik, Universität Frankfurt, Max-von-Laue Strasse 1, 60438 Frankfurt, Germany

March 12, 2020

\begin{abstract}
We discuss collisionless kinetic equations describing the non-equilibrium dynamics of magnons in a ferromagnet exposed to an oscillating microwave field. Previously, this problem has been treated within the so-called "S-theory" where the collision integral in the kinetic equation for the magnon distribution is either neglected or taken into account phenomenologically via an effective relaxation time. However, the possibility of magnon condensation has not been included in S-theory. Moreover, the momentum integrations appearing in the magnon self-energies are usually decoupled by retaining only the term where the loop momentum is equal to the external momentum. In this work we critically examine the accuracy of these approximations and develop the proper extensions of S-theory. We show that these extensions can significantly modify the time evolution of the magnon distribution.
\end{abstract}

\section{Introduction}

Magnons in ordered magnets can be excited by exposing the system to an external oscillating microwave field. Due to the periodic modulation of the coupling parameter, energy can be pumped into the system via parametric resonance. The theory of parametric resonance in magnon gases has a long history, starting with pioneering works by Suhl [1] and by Schlömann et al. 2]. A comprehensive theory of parametric resonance in magnon gases was developed in the 1970's by Zakharov, L'vov, and Starobinets 3 who derived and solved non-linear kinetic equations for the diagonal- and off-diagonal magnon distribution functions

$$
\begin{aligned}
& n_{\boldsymbol{k}}(t)=\left\langle a_{\boldsymbol{k}}^{\dagger}(t) a_{\boldsymbol{k}}(t)\right\rangle, \\
& p_{\boldsymbol{k}}(t)=\left\langle a_{-\boldsymbol{k}}(t) a_{\boldsymbol{k}}(t)\right\rangle,
\end{aligned}
$$

within the time-dependent self-consistent Hartree-Fock approximation. Here $a_{\boldsymbol{k}}(t)$ and $a_{\boldsymbol{k}}^{\dagger}(t)$ are the magnon annihilation and creation operators in the Heisenberg picture and $\langle\ldots\rangle$ denotes the non-equilibrium statistical average. The theoretical framework based on the time-dependent self-consistent Hartree-Fock approximation developed by Zakharov et al. 3,4 has been called "S-theory" and has been quite successful to explain experiments probing the non-equilibrium magnon dynamics in ferromagnetic insulators such as yttrium-iron garnet (YIG) 5. Although previously many authors have used S-theory and extensions thereof to study the physics of pumped magnon gases, $5,6,7,8,9,10,11,12,13,14,15,16,17,18,19$ the available theoretical descriptions are still incomplete. In particular, we believe that the following three points deserve further theoretical attention:

\footnotetext{
a e-mail: hahn@itp.uni-frankfurt.de
}

1.) Magnon condensation: In a recent series of experiments the phenomenon of "Bose-Einstein condensation" of magnons in thin films of YIG has been observed [20,21, 22, 23, 24, 25, 26, 27, 28]. Although this phenomenon can be explained using the classical stochastic Landau-LifshitzGilbert equation, 29] a microscopic quantum mechanical description of the experiments based on a kinetic equation for the magnon distribution including proper collision integrals has not been achieved. In such an approach the original spin model should be bosonized using the Holstein-Primakoff transformation 30 and the kinetic equations for magnon distribution derived from the effective boson model should be solved together with the equation of motion of the expectation values of the magnon annihilation and creation operators 31]

$$
\psi_{\boldsymbol{k}}(t)=\left\langle a_{\boldsymbol{k}}(t)\right\rangle, \quad \psi_{\boldsymbol{k}}^{*}(t)=\left\langle a_{\boldsymbol{k}}^{\dagger}(t)\right\rangle .
$$

However, in this case the distribution functions $n_{\boldsymbol{k}}(t)$ and $p_{\boldsymbol{k}}(t)$ contain redundant information and one should consider instead their connected counter-parts [17,32]

$$
\begin{aligned}
n_{\boldsymbol{k}}^{c}(t) & =\left\langle\delta a_{\boldsymbol{k}}^{\dagger}(t) \delta a_{\boldsymbol{k}}(t)\right\rangle \\
& =\left\langle a_{\boldsymbol{k}}^{\dagger}(t) a_{\boldsymbol{k}}(t)\right\rangle-\left\langle a_{\boldsymbol{k}}^{\dagger}(t)\right\rangle\left\langle a_{\boldsymbol{k}}(t)\right\rangle, \\
p_{\boldsymbol{k}}^{c}(t) & =\left\langle\delta a_{-\boldsymbol{k}}(t) \delta a_{\boldsymbol{k}}(t)\right\rangle \\
& =\left\langle a_{-\boldsymbol{k}}(t) a_{\boldsymbol{k}}(t)\right\rangle-\left\langle a_{-\boldsymbol{k}}(t)\right\rangle\left\langle a_{\boldsymbol{k}}(t)\right\rangle,
\end{aligned}
$$

where $\delta a_{\boldsymbol{k}}(t)=a_{\boldsymbol{k}}(t)-\left\langle a_{\boldsymbol{k}}(t)\right\rangle$. In conventional S-theory [3] the expectation values $\left\langle a_{\boldsymbol{k}}(t)\right\rangle$ and $\left\langle a_{\boldsymbol{k}}^{\dagger}(t)\right\rangle$ are not explicitly taken into account, so that the kinetic equations of S-theory should be modified if the expectation values of the magnon operators are finite. In this work we show how the kinetic equations of S-theory should be modified in the presence of magnon condensation.

2.) Mode-decoupling: If the collision integrals in the kinetic equations for the magnon distribution are completely 
neglected, S-theory reduces to the time-dependent selfconsistent Hartree-Fock approximation. Interaction effects are then taken into account via a time-dependent magnon self-energy $\Sigma_{\boldsymbol{k}}(t)$ which depends on the distribution function $n_{\boldsymbol{q}}(t)$ for all momenta $\boldsymbol{q}$,

$$
\Sigma_{\boldsymbol{k}}(t)=\frac{1}{N} \sum_{\boldsymbol{q}} T_{\boldsymbol{k}, \boldsymbol{q}} n_{\boldsymbol{q}}(t)
$$

where the $T_{\boldsymbol{k}, \boldsymbol{q}}$ is a matrix element of the two-magnon interaction vertex (see Eq. 25a) below) and $N$ is the number of lattice sites. The resulting kinetic equation is then an integro-differential equation, which will be discussed in detail in Sect. 3. To reduce the mathematical complexity of the problem, it has been proposed $3,4,6$, to retain only the term with $\boldsymbol{q}=\boldsymbol{k}$ in the sum of Eq. (4), which amounts to replacing the self-consistent Hartree-Fock self-energy by

$$
\Sigma_{\boldsymbol{k}}(t) \rightarrow \frac{1}{N} T_{\boldsymbol{k}, \boldsymbol{k}} n_{\boldsymbol{k}}(t) .
$$

The authors of Refs. [3, 4,6] have argued that this decoupling of modes is justified to describe a stationary state where only the mode with the smallest damping (which was introduced phenomenologically) is significantly populated. If the damping of all other modes is sufficiently strong, their contribution to the sum (4) can be neglected for long times, so that only a single term with the smallest damping survives. Although Zahkarov et al. 3, 4, 4, have given intuitive arguments how the damping selects the appropriate mode leading to Eq. (5), it is somewhat unsatisfying that the damping was introduced phenomenologically. If the damping indeed has the assumed form, the resulting stationary magnon distribution is a reasonable approximation to the stationary non-equilibrium distribution. On the other hand, the mode decoupling assumed in Eq. (5) cannot be justified to describe the magnon kinetics at finite times. Indeed, we will show in Sect. 5 that the substitution (5) does not give a quantitatively accurate description of the time evolution of the magnon distribution.

3.) Microscopic collision integral: Although the collision integral in the kinetic equation for the magnon distribution has been written down in the Born approximation [8], and the effect of collisions on the non-equilibrium magnon dynamics has been taken into account phenomenologically by introducing (by hand) a relaxation rate into the kinetic equations of S-theory, we have not been able to find in the literature an explicit solution of the quantum kinetic equation for the magnon distribution including the microscopic expressions for the collision integrals. This technically extremely challenging problem is beyond the scope of this work 33 . The collision integrals are of a more complex form than the self-energy $\Sigma_{\boldsymbol{k}}(t)$ given by Eq. (4) as they involve two momentum integrations and depend on the non-equilibrium distribution functions. In the stationary non-equilibrium state the explicit calculation of the collision integrals is therefore numerically very challenging. It turns out that the microscopic collision integrals as a function of the external magnetic field strength display peaks at certain field strengths leading to enhanced or reduced magnon densities 33 .

The rest of this article is organized as follows. In Sect. 2 we briefly summarize the derivation of a microscopic boson Hamiltonian describing the pumped magnon gas in a ferromagnetic insulator such as YIG. In Sect. 3 we derive the kinetic equations describing the time-evolution of the magnon distribution using different levels of approximation. In particular, we derive the modifications of S-theory in the presence of magnon condensation. The stationary non-equilibrium solution of these equations are derived in Sect. 4. while in Sect. 5 the resulting time-evolution of the magnon distribution is calculated numerically to assess the effect of magnon condensation and the effect of the mode-decoupling approximation (4) on the timeevolution of the distribution function within S-theory. Our main results are summarized in Sect. 6. To make our work self-contained, we describe in the appendix the derivation of the effective magnon Hamiltonian and the two-magnon interaction vertices specifically for YIG.

\section{Hamiltonian for pumped magnons in YIG}

To set up our notation, let us briefly outline the derivation of the effective Hamiltonian describing the pumped magnon gas in YIG starting from the time-dependent spin Hamiltonian [5, 14, 16, 17, 34, 35, 36, 37

$$
\begin{aligned}
\mathcal{H}(t)= & -\frac{1}{2} \sum_{i j} \sum_{\alpha \beta}\left[J_{i j} \delta^{\alpha \beta}+D_{i j}^{\alpha \beta}\right] S_{i}^{\alpha} S_{j}^{\beta} \\
& -\left[h_{0}+h_{1} \cos \left(\omega_{0} t\right)\right] \sum_{i} S_{i}^{z},
\end{aligned}
$$

where $i, j=1, \ldots, N$ label the sites $\boldsymbol{r}_{i}$ and $\boldsymbol{r}_{j}$ of a cubic lattice with $N$ sites and lattice spacing $a \approx 12.376 \AA$, and $\alpha, \beta=x, y, z$ denote to the three components of the spin operators $S_{i}^{\alpha}$. The exchange coupling $J_{i j}=J\left(\boldsymbol{r}_{i}-\boldsymbol{r}_{j}\right)$ between nearest neighbors has the numerical value $J \approx$ $1.29 \mathrm{~K}$. The energy scales $h_{0}=\mu H_{0}$ and $h_{1}=\mu H_{1}$ (where $\left.\mu=g \mu_{B}\right)$ represent the Zeeman energies associated with a static magnetic field $H_{0}$ and a time dependent field $H_{1}$ oscillating with frequency $\omega_{0}$. As explained in Refs. 37, 38 , we may set the $g$-factor equal to two and work with an effective spin $S \approx 14.2$. We restrict ourselves to the description of an infinitely long stripe aligned with the $z$ axis of width $w$ and thickness $d=N a$. For experimentally relevant YIG stripes this thickness is several thousand lattice spacings. The dipolar tensor $D_{i j}^{\alpha \beta}=D^{\alpha \beta}\left(\boldsymbol{r}_{i}-\boldsymbol{r}_{j}\right)$ can be written as 37, 40

$$
D_{i j}^{\alpha \beta}=\left(1-\delta_{i j}\right) \frac{\mu^{2}}{\left|\boldsymbol{r}_{i j}\right|^{3}}\left[3 \hat{r}_{i j}^{\alpha} \hat{r}_{i j}^{\beta}-\delta^{\alpha \beta}\right],
$$

where $\boldsymbol{r}_{i j}=\boldsymbol{r}_{i}-\boldsymbol{r}_{j}$ and $\hat{\boldsymbol{r}}_{i j}=\boldsymbol{r}_{i j} /\left|\boldsymbol{r}_{i j}\right|$. We assume that the classical ground state is a saturated ferromagnet for magnetic fields oriented along the direction of the stripe. In the ground state all spins align with the static magnetic 
field which defines the direction of the macroscopic magnetization. The spin Hamiltonian (6) can then be bosonized via the Holstein-Primakoff transformation [30] and the resulting effective boson Hamiltonian can be expanded in powers of the small parameter $1 / S$,

$$
\mathcal{H}(t)=\mathcal{H}_{0}(t)+\mathcal{H}_{2}(t)+\mathcal{H}_{3}+\mathcal{H}_{4}+\mathcal{O}\left(S^{-1 / 2}\right),
$$

where the $\mathcal{H}_{n}$ contain all terms of order $n$ in the boson operators. We apply a partial Fourier transformation in the $y z$-plane assuming the width $w$ of the sample is infinite, which is reasonable because for experimentally relevant geometries the width $w$ is much larger than the thickness d. As explained in the appendix (see also Refs. [17, 34 37.) the time-independent part of the off-diagonal terms in the quadratic part $\mathcal{H}_{2}(t)$ of the effective boson Hamiltonian are then eliminated by means of a Bogoliubov transformation and the non-resonant terms in the remaining time-dependent part of $\mathcal{H}_{2}(t)$ are simply dropped. With these approximations

$$
\begin{array}{r}
\mathcal{H}_{2}(t)=\sum_{\boldsymbol{k}}\left[\varepsilon_{\boldsymbol{k}} a_{\boldsymbol{k}}^{\dagger} a_{\boldsymbol{k}}\right. \\
+\frac{1}{2} V_{\boldsymbol{k}} \mathrm{e}^{-i \omega_{0} t} a_{\boldsymbol{k}}^{\dagger} a_{-\boldsymbol{k}}^{\dagger} \\
\left.+\frac{1}{2} V_{\boldsymbol{k}}^{*} \mathrm{e}^{i \omega_{0} t} a_{-\boldsymbol{k}} a_{\boldsymbol{k}}\right]
\end{array}
$$

where the boson operator $a_{k}^{\dagger}$ creates a magnon with energy $\varepsilon_{\boldsymbol{k}}$. In the long wavelength limit $\varepsilon_{\boldsymbol{k}}$ can be approximated by $37,38,39$

$$
\varepsilon_{\boldsymbol{k}}=\sqrt{\left[h_{0}+\rho \boldsymbol{k}^{2}+\left(1-f_{\boldsymbol{k}}\right) \Delta \sin ^{2} \theta_{\boldsymbol{k}}\right]\left[h_{0}+\rho \boldsymbol{k}^{2}+f_{\boldsymbol{k}} \Delta\right]},
$$

where the spin stiffness associated with the exchange energy is denoted by $\rho=J S a^{2}$, and the energy scale associated with the dipolar energy is $\Delta=4 \pi \mu^{2} S / a^{3}$. The inplane wavevector $\boldsymbol{k}$ is parametrized as $\boldsymbol{k}=k_{z} \boldsymbol{e}_{z}+k_{y} \boldsymbol{e}_{y}=$ $|\boldsymbol{k}|\left(\cos \theta_{\boldsymbol{k}} \boldsymbol{e}_{z}+\sin \theta_{\boldsymbol{k}} \boldsymbol{e}_{y}\right)$, so that $\theta_{\boldsymbol{k}}$ is the angle between the in-plane wavevector $\boldsymbol{k}$ and the external magnetic field. The form factor $f_{\boldsymbol{k}}$ can be approximated by 37

$$
f_{\boldsymbol{k}}=\frac{1-\mathrm{e}^{-|\boldsymbol{k}| d}}{|\boldsymbol{k}| d}
$$

and the pumping energy $V_{\boldsymbol{k}}$ is $[17,34,35$

$$
V_{\boldsymbol{k}}=\frac{h_{1} \Delta}{4 \varepsilon_{\boldsymbol{k}}}\left[-f_{\boldsymbol{k}}+\left(1-f_{\boldsymbol{k}}\right) \sin ^{2} \theta_{\boldsymbol{k}}\right]
$$

We can remove the explicit time-dependence of $\mathcal{H}_{2}(t)$ in Eq. (9) by transforming to the rotating reference frame via the canonical transformation

$$
\tilde{a}_{\boldsymbol{k}}=\mathrm{e}^{i \frac{\omega_{0}}{2} t} a_{\boldsymbol{k}}, \quad \tilde{a}_{\boldsymbol{k}}^{\dagger}=\mathrm{e}^{-i \frac{\omega_{0}}{2} t} a_{\boldsymbol{k}}^{\dagger} .
$$

The quadratic part of the transformed Hamiltonian in the rotating reference frame is then independent of time,

$$
\tilde{\mathcal{H}}_{2}=\sum_{\boldsymbol{k}}\left[E_{\boldsymbol{k}} \tilde{a}_{\boldsymbol{k}}^{\dagger} \tilde{a}_{\boldsymbol{k}}+\frac{V_{\boldsymbol{k}}}{2} \tilde{a}_{\boldsymbol{k}}^{\dagger} \tilde{a}_{-\boldsymbol{k}}^{\dagger}+\frac{V_{\boldsymbol{k}}^{*}}{2} \tilde{a}_{-\boldsymbol{k}} \tilde{a}_{\boldsymbol{k}}\right],
$$

with shifted magnon energy $E_{\boldsymbol{k}}=\varepsilon_{\boldsymbol{k}}-\omega_{0} / 2$. Unfortunately, the canonical transformation (13) generates some explicit time-dependence in the interaction part of the Hamiltonian 34. For example, the cubic part $\tilde{\mathcal{H}}_{3}(t)$ of the magnon Hamiltonian contains terms of the form $e^{i \omega_{0} t / 2} \tilde{a}_{-\boldsymbol{k}_{1}}^{\dagger} \tilde{a}_{-\boldsymbol{k}_{2}}^{\dagger} \tilde{a}_{\boldsymbol{k}_{1}+\boldsymbol{k}_{2}}$. For our purpose we can simply neglect $\tilde{\mathcal{H}}_{3}(t)$ because it involves only rapidly oscillating terms with frequency $\omega_{0} / 2 \gg\left|\epsilon_{\boldsymbol{k}}-\omega_{0} / 2\right|$ which should be consistently dropped in rotating-wave approximation. Finally, the quartic part of the magnon Hamiltonian in the rotating reference frame is of the form 34

$$
\begin{aligned}
\tilde{\mathcal{H}}_{4}(t)= & \frac{1}{N} \sum_{\boldsymbol{k}_{1}, \ldots, \boldsymbol{k}_{4}} \delta_{\boldsymbol{k}_{1}+\cdots+\boldsymbol{k}_{4}, 0}\left[\frac{1}{(2 !)^{2}} \Gamma_{1,2 ; 3,4}^{\bar{a} \bar{a} a a} \tilde{a}_{-1}^{\dagger} \tilde{a}_{-2}^{\dagger} \tilde{a}_{3} \tilde{a}_{4}\right. \\
& +\frac{1}{3 !} e^{-i \omega_{0} t} \Gamma_{1 ; 2,3,4}^{\bar{a} a a a} \tilde{a}_{-1}^{\dagger} \tilde{a}_{2} \tilde{a}_{3} \tilde{a}_{4} \\
& +\frac{1}{3 !} e^{i \omega_{0} t} \Gamma_{1,2,3 ; 4}^{\bar{a} \bar{a} \bar{a} a} \tilde{a}_{-1}^{\dagger} \tilde{a}_{-2}^{\dagger} \tilde{a}_{-3}^{\dagger} \tilde{a}_{4} \\
& +\frac{1}{4 !} e^{-2 i \omega_{0} t} \Gamma_{1,2,3,4}^{a a a a} \tilde{a}_{1} \tilde{a}_{2} \tilde{a}_{3} \tilde{a}_{4} \\
& \left.+\frac{1}{4 !} e^{2 i \omega_{0} t} \Gamma_{1,2,3,4}^{\bar{a} \bar{a} \bar{a} \bar{a}} \tilde{a}_{-1}^{\dagger} \tilde{a}_{-2}^{\dagger} \tilde{a}_{-3}^{\dagger} \tilde{a}_{-4}^{\dagger}\right]
\end{aligned}
$$

where the Kronecker- $\delta$ enforces momentum conservation and we use the abbreviation $\tilde{a}_{1}=\tilde{a}_{\boldsymbol{k}_{1}}$. Explicit expressions for the two-magnon interaction vertices appearing in Eq. 15 are given in the appendix, see Eqs. (A18aA18e. Within the rotating-wave approximation, it is consistent to drop all oscillating terms on the right-hand side of Eq. (15).

\section{Collisionless kinetic equations}

In this section we derive kinetic equations for the magnon distribution in the rotating reference frame using three different levels of approximation. However, a proper microscopic treatment of the collision integrals is beyond the scope of this work 33. Note that the phase factor $e^{ \pm i \omega_{0} t / 2}$ in Eq. (13) cancels in the diagonal distribution $n_{\boldsymbol{k}}(t)$, so that in the rotating reference frame the diagonal and the off-diagonal distribution functions are given by

$$
\begin{aligned}
& n_{\boldsymbol{k}}(t)=\left\langle\tilde{a}_{\boldsymbol{k}}^{\dagger}(t) \tilde{a}_{\boldsymbol{k}}(t)\right\rangle=\left\langle a_{\boldsymbol{k}}^{\dagger}(t) a_{\boldsymbol{k}}(t)\right\rangle, \\
& \tilde{p}_{\boldsymbol{k}}(t)=\left\langle\tilde{a}_{-\boldsymbol{k}}(t) \tilde{a}_{\boldsymbol{k}}(t)\right\rangle=\mathrm{e}^{i \omega_{0} t} p_{\boldsymbol{k}}(t) .
\end{aligned}
$$

\subsection{Non-interacting system}

To begin with, let us completely neglect all magnon-magnon interactions and approximate the Hamiltonian for the pumped magnon gas in the rotating reference frame by the quadratic Hamiltonian $\tilde{\mathcal{H}}_{2}$ in Eq. (14). From the Heisenberg equations of motion in the rotating reference frame,

$$
i \partial_{t} \tilde{a}_{\boldsymbol{k}}=\left[\tilde{a}_{\boldsymbol{k}}, \tilde{\mathcal{H}}(t)\right], \quad i \partial_{t} \tilde{a}_{\boldsymbol{k}}^{\dagger}=\left[\tilde{a}_{\boldsymbol{k}}^{\dagger}, \tilde{\mathcal{H}}(t)\right]
$$


it is then easy to show that

$$
\begin{aligned}
\partial_{t} n_{\boldsymbol{k}}(t)+i\left[V_{\boldsymbol{k}} \tilde{p}_{\boldsymbol{k}}^{*}(t)-V_{\boldsymbol{k}}^{*} \tilde{p}_{\boldsymbol{k}}(t)\right] & =0, \\
\partial_{t} \tilde{p}_{\boldsymbol{k}}(t)+2 i E_{\boldsymbol{k}} \tilde{p}_{\boldsymbol{k}}(t)+i V_{\boldsymbol{k}}\left[2 n_{\boldsymbol{k}}(t)+1\right] & =0,
\end{aligned}
$$

where in the second equation we have used $E_{\boldsymbol{k}}=E_{-\boldsymbol{k}}$ and $n_{\boldsymbol{k}}=n_{-\boldsymbol{k}}$. These equations can be solved exactly [17. In the regime $\left|E_{\boldsymbol{k}}\right|>\left|V_{\boldsymbol{k}}\right|$ the solutions exhibit an oscillatory behavior, while in the strong-pumping regime where $\left|V_{\boldsymbol{k}}\right|>\left|E_{\boldsymbol{k}}\right|$ the solutions grow exponentially in time [1,2,17.

\subsection{S-theory: time-dependent self-consistent Hartree-Fock approximation}

Interactions between magnons eventually lead to a saturation of the exponential growth of the magnon distribution in the strong-pumping regime. The simplest approximation which includes this interaction-induced saturation mechanism is the time-dependent self-consistent HartreeFock approximation, which in this context is called Stheory [3, 4, 12, 14, 16]. The kinetic equations $(18)$ and $(19)$ are then replaced by

$$
\begin{aligned}
\partial_{t} n_{\boldsymbol{k}}(t)+i\left[\tilde{V}_{\boldsymbol{k}}(t) \tilde{p}_{\boldsymbol{k}}^{*}(t)-\tilde{V}_{\boldsymbol{k}}^{*}(t) \tilde{p}_{\boldsymbol{k}}(t)\right] & =0, \\
\partial_{t} \tilde{p}_{\boldsymbol{k}}(t)+2 i \tilde{E}_{\boldsymbol{k}}(t) \tilde{p}_{\boldsymbol{k}}(t)+i \tilde{V}_{\boldsymbol{k}}(t)\left[2 n_{\boldsymbol{k}}(t)+1\right] & =0,
\end{aligned}
$$

where the renormalized magnon dispersion $\tilde{E}_{\boldsymbol{k}}(t)$ and pumping energy $\tilde{V}_{\boldsymbol{k}}(t)$ depend now on the distribution functions,

$$
\begin{aligned}
& \tilde{E}_{\boldsymbol{k}}(t)=E_{\boldsymbol{k}}+\frac{1}{N} \sum_{\boldsymbol{q}}\left[\Gamma_{-\boldsymbol{k},-\boldsymbol{q} ; \boldsymbol{q}, \boldsymbol{k}}^{\bar{a} \bar{a} a} n_{\boldsymbol{q}}(t)\right. \\
& \left.+\frac{1}{2} \mathrm{e}^{-i \omega_{0} t} \Gamma_{-\boldsymbol{k} ;-\boldsymbol{q}, \boldsymbol{q}, \boldsymbol{k}}^{\bar{a} a a a} \tilde{p}_{\boldsymbol{q}}(t)+\frac{1}{2} \mathrm{e}^{i \omega_{0} t} \Gamma_{-\boldsymbol{k},-\boldsymbol{q}, \boldsymbol{q} ; \boldsymbol{k}}^{\bar{a} \bar{a} \bar{a} a} \tilde{p}_{\boldsymbol{q}}^{*}(t)\right], \\
& \tilde{V}_{\boldsymbol{k}}(t)=V_{\boldsymbol{k}}+\frac{1}{2 N} \sum_{\boldsymbol{q}}\left[\mathrm{e}^{i \omega_{0} t} \Gamma_{-\boldsymbol{k}, \boldsymbol{k},-\boldsymbol{q} ; \boldsymbol{q}}^{\bar{a} \bar{a} \bar{a} a} n_{\boldsymbol{q}}(t)\right. \\
& \left.+\frac{1}{2} \Gamma_{-\boldsymbol{k}, \boldsymbol{k} ;-\boldsymbol{q}, \boldsymbol{q}}^{\bar{a} \bar{a} a a} \tilde{p}_{\boldsymbol{q}}(t)+\frac{1}{2} \mathrm{e}^{2 i \omega_{0} t} \Gamma_{-\boldsymbol{k}, \boldsymbol{k},-\boldsymbol{q} ; \boldsymbol{q}}^{\bar{a} \bar{a} \bar{a} \bar{a}} \tilde{p}_{\boldsymbol{q}}^{*}(t)\right] .
\end{aligned}
$$

Within the rotating-wave approximation we can drop the rapidly oscillating terms proportional to $\mathrm{e}^{ \pm i \omega_{0} t}$ and $\mathrm{e}^{ \pm 2 i \omega_{0} t}$, so that the above self-consistency equation reduce to

$$
\begin{aligned}
& \tilde{E}_{\boldsymbol{k}}(t)=E_{\boldsymbol{k}}+\frac{1}{N} \sum_{\boldsymbol{q}} T_{\boldsymbol{k}, \boldsymbol{q}} n_{\boldsymbol{q}}(t), \\
& \tilde{V}_{\boldsymbol{k}}(t)=V_{\boldsymbol{k}}+\frac{1}{2 N} \sum_{\boldsymbol{q}} S_{\boldsymbol{k}, \boldsymbol{q}} \tilde{p}_{\boldsymbol{q}}(t),
\end{aligned}
$$

with

$$
\begin{aligned}
T_{\boldsymbol{k}, \boldsymbol{q}} & =\Gamma_{-\boldsymbol{k},-\boldsymbol{q} ; \boldsymbol{q}, \boldsymbol{k}}^{\bar{a} \bar{a} a a}, \\
S_{\boldsymbol{k}, \boldsymbol{q}} & =\Gamma_{-\boldsymbol{k}, \boldsymbol{k} ;-\boldsymbol{q}, \boldsymbol{q}}^{\bar{a} \bar{a} a a} .
\end{aligned}
$$

\subsection{Mode decoupling}

At this point the kinetic equations are still non-trivial integro-differential equations. However, the authors of Refs. [3, 4, 6] have proposed to decouple the modes with different wavevectors by approximating the sums in Eqs. (24a) and (24b) by the single term where the loop momentum $\boldsymbol{q}$ is equal to the external momentum $\boldsymbol{k}$,

$$
\begin{aligned}
\tilde{E}_{\boldsymbol{k}}(t) & \approx E_{\boldsymbol{k}}+\frac{1}{N} T_{\boldsymbol{k}, \boldsymbol{k}} n_{\boldsymbol{k}}(t), \\
\tilde{V}_{\boldsymbol{k}}(t) & \approx V_{\boldsymbol{k}}+\frac{1}{2 N} S_{\boldsymbol{k}, \boldsymbol{k}} \tilde{p}_{\boldsymbol{k}}(t) .
\end{aligned}
$$

As already mentioned in the introduction after Eq. (4), this substitution has been justified by observing that after sufficiently long times only the mode with the longest lifetime is significantly populated, so that the momentum dependence of the magnon damping is crucial to justify Eq. (26). This substitution can therefore only be used to describe the asymptotic long-time dynamics of the magnon distribution, including a possible stationary non-equilibrium state.

\subsection{S-theory with magnon condensation}

In the previous subsection, we have not taken into account the possibility that the non-equilibrium expectation values $\psi_{\boldsymbol{k}}(t)=\left\langle a_{\boldsymbol{k}}(t)\right\rangle$ of the magnon operators are finite, see Eq. (2). In the strong-pumping regime or in the regime where the magnons condense, the finite value of $\psi_{\boldsymbol{k}}(t)$ cannot be ignored, so that we should complement our system of kinetic equations by an equation of motion for $\psi_{\boldsymbol{k}}(t)$, which is the analogue of the Gross-Pitaevskii equation describing the dynamics of the order parameter of a superfluid. The parametrization of the dynamics in terms of the correlation functions $n_{\boldsymbol{k}}(t)$ and $\tilde{p}_{\boldsymbol{k}}(t)$ is then redundant and it is better to consider their connected counter-parts in the rotating reference frame, 17

$$
\begin{gathered}
n_{\boldsymbol{k}}^{c}(t)=\left\langle\delta \tilde{a}_{\boldsymbol{k}}^{\dagger}(t) \delta \tilde{a}_{\boldsymbol{k}}(t)\right\rangle, \\
\tilde{p}_{\boldsymbol{k}}^{c}(t)=\left\langle\delta \tilde{a}_{-\boldsymbol{k}}(t) \delta \tilde{a}_{\boldsymbol{k}}(t)\right\rangle,
\end{gathered}
$$

where $\delta \tilde{a}_{\boldsymbol{k}}(t)=\tilde{a}_{\boldsymbol{k}}(t)-\left\langle\tilde{a}_{\boldsymbol{k}}(t)\right\rangle$. Note that conventional S-theory does not explicitly take finite expectation values of the magnon annihilation and creation operators into account. Defining the expectation value of the magnon operators in the rotating reference frame,

$$
\tilde{\psi}_{\boldsymbol{k}}(t)=\left\langle\tilde{a}_{\boldsymbol{k}}(t)\right\rangle,
$$

we obtain within the self-consistent time-dependent Hartree-Fock approximation and the rotating-wave approximation,

$$
\begin{aligned}
\partial_{t} n_{\boldsymbol{k}}^{c}+i\left[\tilde{V}_{\boldsymbol{k}}\left(\tilde{p}_{\boldsymbol{k}}^{c}\right)^{*}-\tilde{V}_{\boldsymbol{k}}^{*} \tilde{p}_{\boldsymbol{k}}^{c}\right] & =0, \\
\partial_{t} \tilde{p}_{\boldsymbol{k}}^{c}+2 i \tilde{E}_{\boldsymbol{k}} \tilde{p}_{\boldsymbol{k}}^{c}+i \tilde{V}_{\boldsymbol{k}}\left[2 n_{\boldsymbol{k}}^{c}+1\right] & =0, \\
\partial_{t} \tilde{\psi}_{\boldsymbol{k}}+i \tilde{E}_{\boldsymbol{k}} \tilde{\psi}_{\boldsymbol{k}}+i \tilde{V}_{\boldsymbol{k}} \tilde{\psi}_{-\boldsymbol{k}}^{*} & =0,
\end{aligned}
$$


where it is understood that all quantities are timedependent and the renormalized magnon dispersion and pumping energy are now given by

$$
\begin{aligned}
& \tilde{E}_{\boldsymbol{k}}=E_{\boldsymbol{k}}+\frac{1}{N} \sum_{\boldsymbol{q}} T_{\boldsymbol{k}, \boldsymbol{q}}\left(n_{\boldsymbol{q}}^{c}+\left|\tilde{\psi}_{\boldsymbol{q}}\right|^{2}\right), \\
& \tilde{V}_{\boldsymbol{k}}=V_{\boldsymbol{k}}+\frac{1}{2 N} \sum_{\boldsymbol{q}} S_{\boldsymbol{k}, \boldsymbol{q}}\left(\tilde{p}_{\boldsymbol{q}}^{c}+\tilde{\psi}_{-\boldsymbol{q}} \tilde{\psi}_{\boldsymbol{q}}\right) .
\end{aligned}
$$

\section{Stationary non-equilibrium state}

Within the approximations described in Sect. 3.2 we can find a stationary non-equilibrium solution for the distribution functions. We further simplify the self-consistent energy $\tilde{E}_{\boldsymbol{k}}(t)$ and the pumping $\tilde{V}_{\boldsymbol{k}}(t)$ using the modedecoupling approximation described by Eqs. 26a and (26b). We also approximate $2 n_{\boldsymbol{k}}^{c}+1 \approx 2 n_{\boldsymbol{k}}^{c}$ in Eq. (30) because the magnon density in the stationary state is of order $N \gg 1$. In the regime of parametric instability,

$$
\left|V_{k}\right|>\left|E_{k}\right|
$$

we find the stationary solution within S-theory [3, 4,

$$
\begin{aligned}
& n_{\boldsymbol{k}}=N \frac{\left|V_{\boldsymbol{k}}\right|-\left|E_{\boldsymbol{k}}\right|}{T_{\boldsymbol{k}, \boldsymbol{k}}+\frac{1}{2} S_{\boldsymbol{k}, \boldsymbol{k}}}, \\
& \tilde{p}_{\boldsymbol{k}}=-n_{\boldsymbol{k}} .
\end{aligned}
$$

Eq. (33) implies that there is a minimum strength of the pumping field $h_{1}$ which is necessary to macroscopically populate magnons with wavevector $\boldsymbol{k}$ and energy $\varepsilon_{\boldsymbol{k}}$,

$$
h_{1, \min }(\boldsymbol{k})=\frac{4}{\Delta}\left|\frac{\varepsilon_{\boldsymbol{k}}\left(\varepsilon_{\boldsymbol{k}}-\frac{\omega_{0}}{2}\right)}{-f_{\boldsymbol{k}}+\left(1-f_{\boldsymbol{k}}\right) \sin ^{2} \theta_{\boldsymbol{k}}}\right| .
$$

Note that at this point we have neglected magnon damping which would lead to a relaxation of magnon occupation. Without damping, this equation suggests that magnons satisfying $\epsilon_{\boldsymbol{k}}=\omega_{0} / 2$ are always excited for infinitesimal small pumping fields $H_{1}$.

The above results for the stationary magnon distributions do not change if we use the modified S-theory including magnon condensation as described in Sect. 3.4. In this case we obtain from (29)-(31) with $\partial_{t} n_{\boldsymbol{k}}^{c}=\partial_{t} \tilde{p}_{\boldsymbol{k}}^{c}=$ $\partial_{t} \tilde{\psi}_{\boldsymbol{k}}=0$,

$$
\begin{aligned}
& n_{\boldsymbol{k}}=n_{\boldsymbol{k}}^{c}+\left|\tilde{\psi}_{\boldsymbol{k}}\right|^{2}=N \frac{\left|V_{\boldsymbol{k}}\right|-\left|E_{\boldsymbol{k}}\right|}{T_{\boldsymbol{k}, \boldsymbol{k}}+\frac{1}{2} S_{\boldsymbol{k}, \boldsymbol{k}}}, \\
& \tilde{p}_{\boldsymbol{k}}=\tilde{p}_{\boldsymbol{k}}^{c}+\left(\tilde{\psi}_{\boldsymbol{k}}\right)^{2}=-n_{\boldsymbol{k}}^{c}-\left|\tilde{\psi}_{\boldsymbol{k}}\right|^{2} .
\end{aligned}
$$

Note that the partition of the magnon distribution function $n_{\boldsymbol{k}}$ into a connected part $n_{\boldsymbol{k}}^{c}$ and a contribution $\left|\tilde{\psi}_{\boldsymbol{k}}\right|^{2}$ from the finite expectation value of the magnon operators is ambiguous as long as the magnon damping is neglected.
Another way to derive the stationary magnon distribution functions in Eqs. (34) is to assume that in the stationary state only one pair of magnons with momenta $\pm \boldsymbol{k}$ is significantly occupied. This assumption was justified in Refs. 3, 4] by invoking again the phenomenologically introduced magnon damping, which leads to a decoupling of magnon modes. If we initially prepare the system in a state where more than one pair of $\boldsymbol{k}$ modes is significantly occupied, the usual argument is that after sufficiently long times only the pair of modes with the smallest damping will survive. A simple phenomenological way to model the effect of magnon damping is by inserting (by hand) a damping rate $\gamma_{\boldsymbol{k}}$ into the equations of motion of the magnon creation and annihilation operators. In the rotating reference frame the resulting modified equations of motion are

$$
\begin{aligned}
& \partial_{t} \tilde{a}_{\boldsymbol{k}}(t)=\left(-i E_{\boldsymbol{k}}-\gamma_{\boldsymbol{k}}\right) \tilde{a}_{\boldsymbol{k}}-i V_{\boldsymbol{k}} \tilde{a}_{-\boldsymbol{k}}^{\dagger}, \\
& \partial_{t} \tilde{a}_{\boldsymbol{k}}^{\dagger}(t)=\left(i E_{\boldsymbol{k}}-\gamma_{\boldsymbol{k}}\right) \tilde{a}_{\boldsymbol{k}}^{\dagger}+i V_{\boldsymbol{k}}^{*} \tilde{a}_{-\boldsymbol{k}} .
\end{aligned}
$$

For simplicity let us focus on the magnons satisfying the resonance condition $\varepsilon_{\boldsymbol{k}}=\omega_{0} / 2$ where $E_{\boldsymbol{k}}=0$. Then Stheory gives a stationary solution [3, 4

$$
\begin{aligned}
& n_{\boldsymbol{k}}=N \frac{\sqrt{V_{\boldsymbol{k}}^{2}-\gamma_{\boldsymbol{k}}^{2}}}{T_{\boldsymbol{k}, \boldsymbol{k}}+\frac{1}{2} S_{\boldsymbol{k}, \boldsymbol{k}}}, \\
& \tilde{p}_{\boldsymbol{k}}=-n_{\boldsymbol{k}},
\end{aligned}
$$

provided the pumping compensates the losses due to damping,

$$
\left|V_{\boldsymbol{k}}\right|>\left|\gamma_{\boldsymbol{k}}\right| \text {. }
$$

In this case a non-zero minimum strength of the pumping field $h_{1, \min }(\boldsymbol{k})>0$ is necessary to obtain a stationary state.

To visualize the magnon distribution in momentum space we discretize the momenta as follows: First of all, we retain only low-energy momenta where $E_{\boldsymbol{k}}=\varepsilon_{\boldsymbol{k}}-\omega_{0} / 2 \in$ $\left[-0.1 \omega_{0}, 0.1 \omega_{0}\right]$. This interval is then subdivided into $N_{\varepsilon}$ shells $\left[\varepsilon_{i}, \varepsilon_{i+1}\right]$ and the wavevectors $\boldsymbol{k}$ with $E_{\boldsymbol{k}} \in\left[\varepsilon_{i}, \varepsilon_{i+1}\right]$ are parametrized by $N_{\alpha}$ discrete angles $\alpha_{j}$ in the interval $[0, \pi / 2]$ as illustrated in Fig. 1

From the plot of the magnon distribution function obtained within S-theory in Fig. 2 (a) we see that for small strength of the pumping field $h_{1} \ll h_{0}$ the instability condition (33) is fulfilled only in a small region of the momentum space near the resonance surface defined by $\varepsilon_{\boldsymbol{k}}=\omega_{0} / 2$. This is also shown in Fig. 2 (b) where the magnon distribution function is plotted over $k_{y}$ for $k_{z}=0$. The shape of the distribution function is mostly determined by the numerator of Eq. (36a), where $V_{\boldsymbol{k}}$ is almost constant along the $y$-axis and the magnon energy depends approximately quadratically on the wavevector. The width of the distribution function is three orders of magnitude smaller than the momentum $k_{0}=\left|\boldsymbol{k}_{0}\right|$ on the resonance surface. This allows us to neglect the nonvanishing magnon distribution function for momenta $k$ which do not satisfy the resonance condition $\epsilon_{\boldsymbol{k}}=\omega_{0} / 2$. It is therefore sufficient to take into account only momenta $\boldsymbol{k}$ with $\varepsilon_{\boldsymbol{k}}=\omega_{0} / 2$ for calculating the stationary 


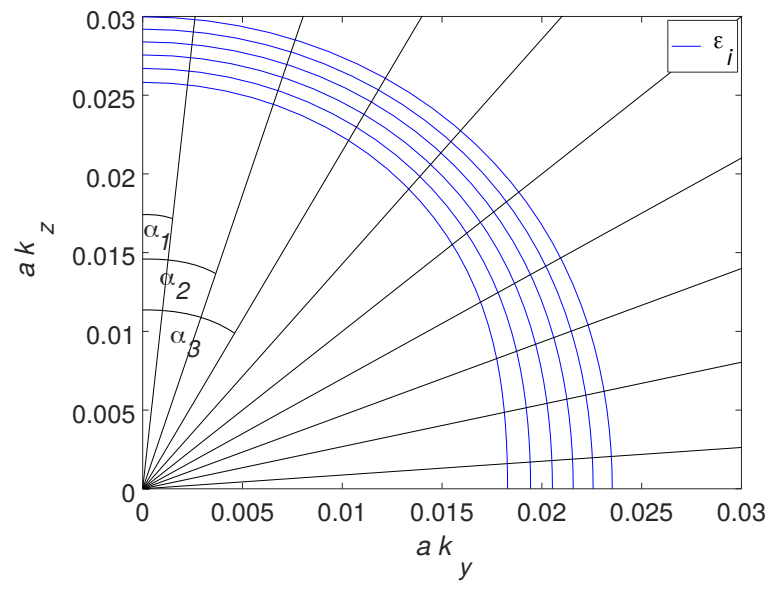

Fig. 1. The discretization of momenta used for the numerical solution of the kinetic equations. $N_{\epsilon}=5$ energy shells with $E_{\boldsymbol{k}} \in\left[-0.1 \omega_{0}, 0.1 \omega_{0}\right]$ are shown in the momentum space. They are further subdivided by $N_{\alpha}=10$ discrete angles in the interval $[0, \pi / 2]$. The wavevectors in the center of the meshes are being used for the further calculations.

non-equilibrium state. To quantitatively examine the accuracy of the mode-decoupling substitution (26) without damping, let us try to construct a stationary solution of Eq. (30) assuming $\tilde{p}_{\boldsymbol{k}}=-n_{\boldsymbol{k}}$. In this case Eq. (30) reduces to the linear integral equation

$$
\frac{1}{N} \sum_{\boldsymbol{q}}\left(T_{\boldsymbol{k}, \boldsymbol{q}}+\frac{1}{2} S_{\boldsymbol{k}, \boldsymbol{q}}\right) n_{\boldsymbol{q}}=\left|V_{\boldsymbol{k}}\right|-E_{\boldsymbol{k}}
$$

Obviously, a unique solution exists only if the matrix $M_{\boldsymbol{k}, \boldsymbol{q}}=T_{\boldsymbol{k}, \boldsymbol{q}}+\frac{1}{2} S_{\boldsymbol{k}, \boldsymbol{q}}$ is invertible. Numerically, we find that in certain parameter regimes this is not the case. Moreover, even in a regime where this matrix is invertible, it can happen that the resulting stationary distribution is negative, which is obviously unphysical. These problems do not arise if we retain only the diagonal elements of the matrix $M_{\boldsymbol{k}, \boldsymbol{q}}$ in which case we recover Eq. (34a). Fig. 3 shows $M_{\boldsymbol{k}, \boldsymbol{q}}$ plotted over the angles $\theta_{\boldsymbol{k}}$ and $\theta_{\boldsymbol{q}}$ of two vectors $\boldsymbol{k}$ and $\boldsymbol{q}$ with $\epsilon_{\boldsymbol{k}}=\epsilon_{\boldsymbol{q}}=\omega_{0} / 2$. A peak for $\boldsymbol{k}=\boldsymbol{q}$ can be observed but this finding is not sufficient to justify the mode decoupling. However, as the stationary solution obtained from the integral equation 40 can have unphysical features, we conclude that the kinetic equations derived in Sect. 3 are not suitable to describe experimentally relevant systems and the replacement of the Hartree-Fock self-energies in Eq. (24) by the decoupled expressions in Eq. (26) is questionable although it gives reasonable results. On the other hand, when taking into account the magnon damping phenomenologically this decoupling can be justified by the mode-selective effect of magnon damping. Thus, when investigating parametrically pumped magnon gases, magnon damping should be accounted for. (a)
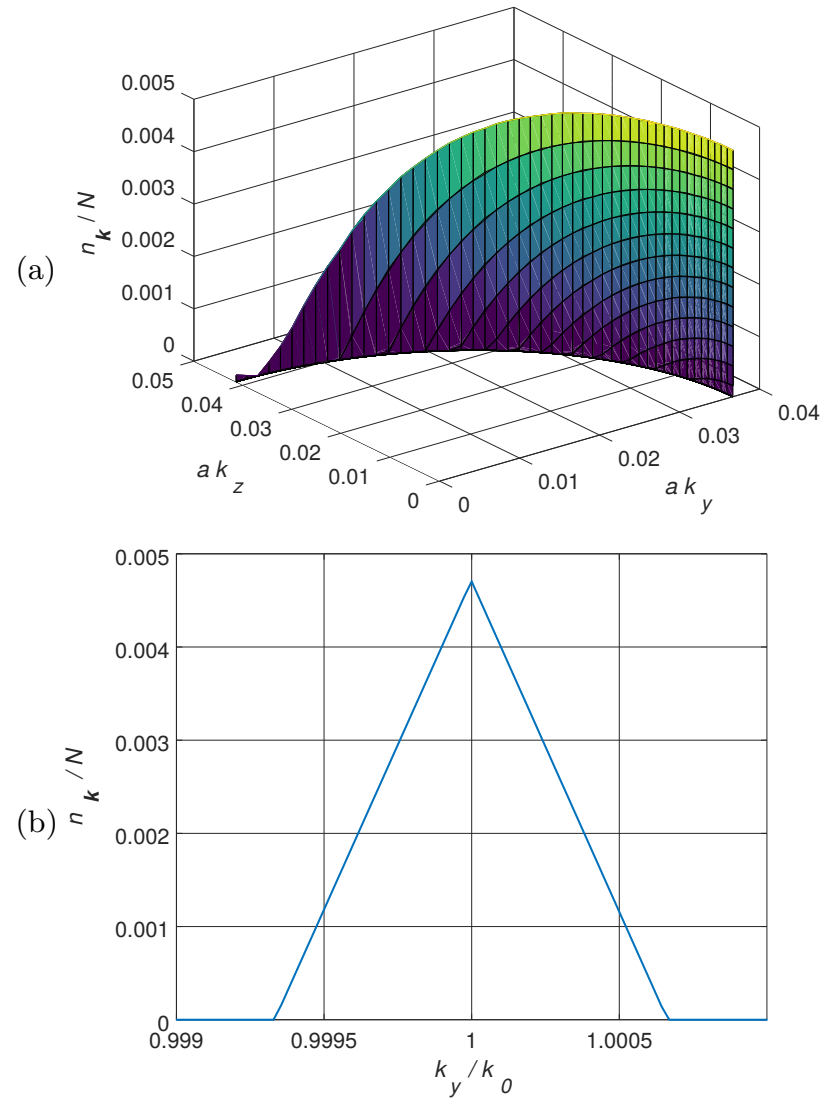

Fig. 2. (a) Plot of the magnon distribution in the stationary solution 34a within S-theory for a thin film of YIG with thickness $d=10 \mu \mathrm{m}$ corresponding to $N=8080$ and $H_{0}=800$ Oe, $H_{1}=40 \mathrm{Oe}$, and $\omega_{0}=25 \mathrm{GHz}$ as a function of both components $k_{y}$ and $k_{z}$ of the in-plane momentum $\boldsymbol{k}$, (b) as a function of $k_{y}$ for $k_{z}=0$, where $k_{0}=\left|\boldsymbol{k}_{0}\right|$ is defined by the resonance condition $\varepsilon_{\boldsymbol{k}_{0}}=\omega_{0} / 2$. The momenta are discretized as shown in Fig. 1 but for different parameters. Note that the magnon density is finite only in a small area around the resonance surface where the condition $\sqrt{33}$ is fulfilled.

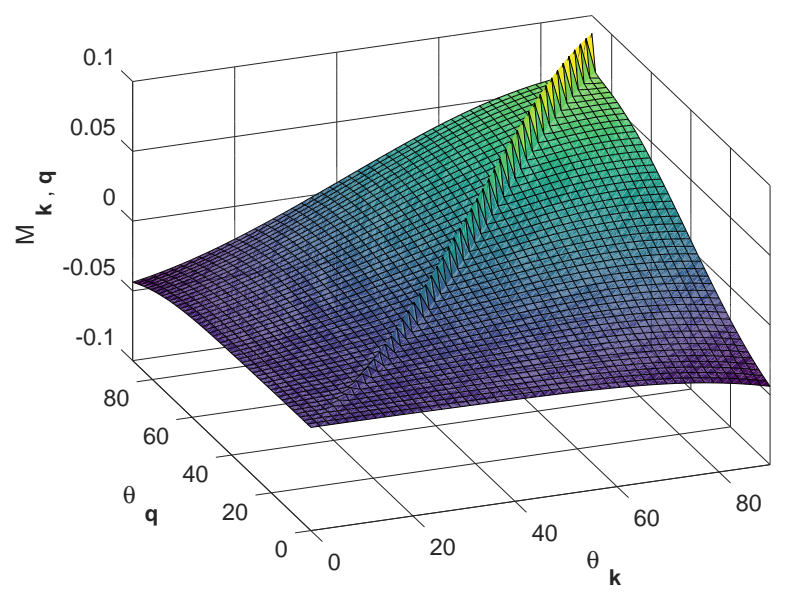

Fig. 3. The dependence of $M_{\boldsymbol{k}, \boldsymbol{q}}=T_{\boldsymbol{k}, \boldsymbol{q}}+S_{\boldsymbol{k}, \boldsymbol{q}} / 2$ on the angles $\theta_{\boldsymbol{k}}$ and $\theta_{\boldsymbol{q}}$ of the two wave vectors $\boldsymbol{k}$ and $\boldsymbol{q}$, where $\boldsymbol{k}$ and $\boldsymbol{q}$ both fulfill the resonance condition $\epsilon_{\boldsymbol{k}}=\epsilon_{\boldsymbol{q}}=\omega_{0} / 2$. 


\section{Collisionless magnon dynamics}

In this section we present numerical results for the time evolution of the magnon distribution obtained from the solution of the collisionless kinetic equations (29)-(31) of modified S-theory with magnon condensation and mode coupling and compare the results with the predictions of conventional S-theory discussed in Sect. 3.2 For the numerical solution of the kinetic equations we discretize the momenta in the same way as above, shown in Fig. 1. The total magnon number and off-diagonal occupation in the rotating reference frame are then approximated by

$$
\begin{aligned}
& n=\sum_{\boldsymbol{k}} n_{\boldsymbol{k}} \approx \sum_{i=1}^{N_{\varepsilon}} \sum_{j=1}^{N_{\alpha}} n_{\boldsymbol{k}_{i j}}, \\
& \tilde{p}=\sum_{\boldsymbol{k}} \tilde{p}_{\boldsymbol{k}} \approx \sum_{i=1}^{N_{\varepsilon}} \sum_{j=1}^{N_{\alpha}} \tilde{p}_{\boldsymbol{k}_{i j}},
\end{aligned}
$$

where $\boldsymbol{k}_{i j}$ is a wavevector in the center of the mesh-points. Our numerical results for the total magnon density $n / N$ and the imaginary part of the off-diagonal density $\operatorname{Im} \tilde{p} / N$ are shown in Fig. 4, where we compare these quantities for the three different versions of S-theory developed in Sect. 3. The numerical results are obtained for a thin YIG film with thickness $d=10 \mu \mathrm{m}$, exposed to a static magnetic field $H_{0}=800$ Oe and oscillating field with amplitude $H_{1}=50$ Oe and frequency $\omega_{0}=13.857 \mathrm{GHz}$. In Fig. 4 (a) we show the time evolution of the total magnon density $n / N$ and of $\operatorname{Im} \tilde{p} / N$ as predicted by the conventional S-theory and mode decoupling as discussed in Sect. 3.3. Because the damping is neglected, the magnon density does not approach a stationary limit but continues to oscillate around the value $n / N=0.46$ which agrees with the stationary value $n=\sum_{k} n_{k}$ obtained from Eq. (34a). Note that in this approximation modes with different wavevectors are completely decoupled. In Fig. 5. (a) we show the time evolution of the occupation number of magnon modes for some representative wavevectors. Due to the mode-decoupling, the occupations oscillate with given characteristic frequencies and amplitudes; summing over all wavevectors we recover the time evolution of the total densities shown in Fig. 4. Fig. 6 shows the Fourier transform of the magnon density which has peaks for low frequencies. As the amplitudes of the modes vary greatly, the time evolution of the total magnon density is dominated by the modes satisfying the parametric resonance condition $\left|V_{\boldsymbol{k}}\right|>\left|E_{\boldsymbol{k}}\right|$. If we completely neglect magnonmagnon interactions, then the magnon occupation grows exponentially in this regime; the Hartree-Fock correlations retained within S-theory cut off the exponential growth and eventually lead to an oscillatory behavior with time average given by the stationary distribution derived in Eq. (34a).

The above results rely on the mode-decoupling approximation 26 . If we do not use this approximation but retain all modes in integrals of the self-energy contributions to the renormalized magnon energy $\tilde{E}_{\boldsymbol{k}}(t)$ and the pumping energy $\tilde{V}_{\boldsymbol{k}}(t)$ defined in Eq. 24 of Sect. 3.2 , the time (a)

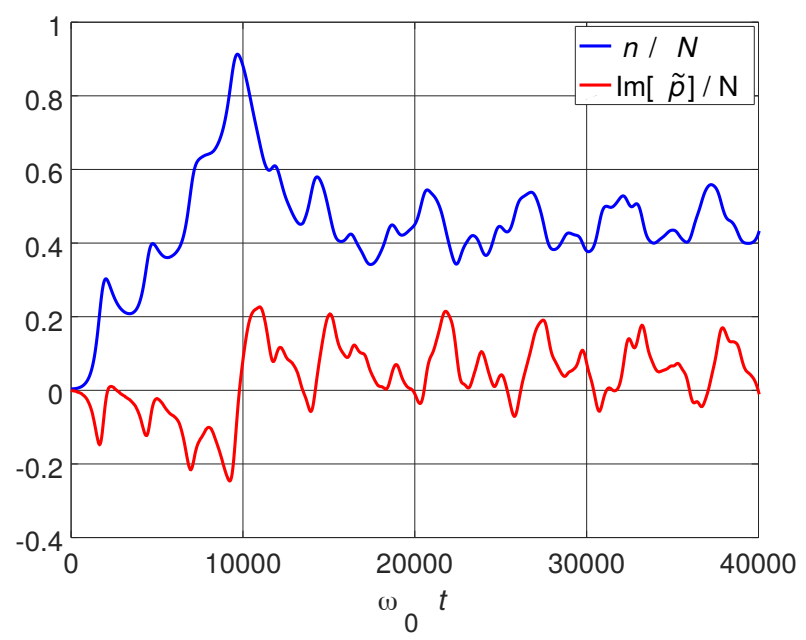

(b)

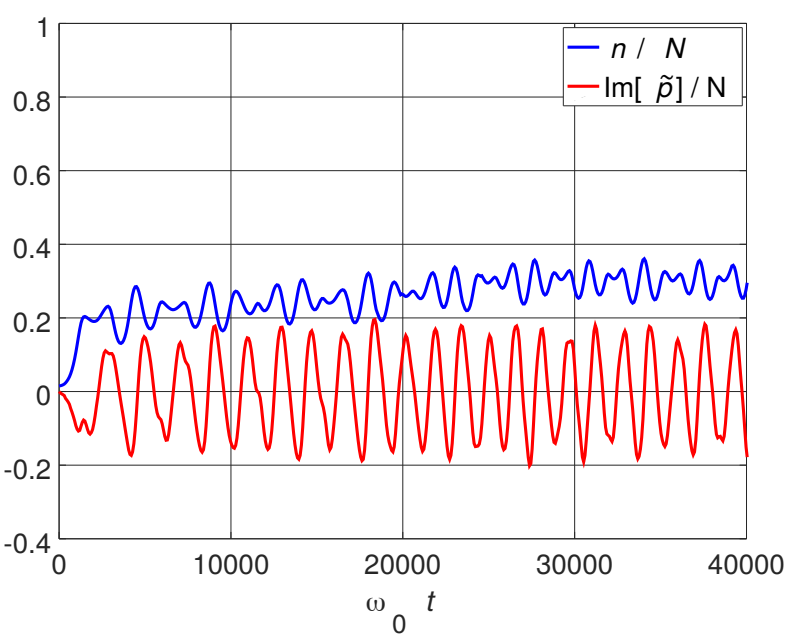

(c)
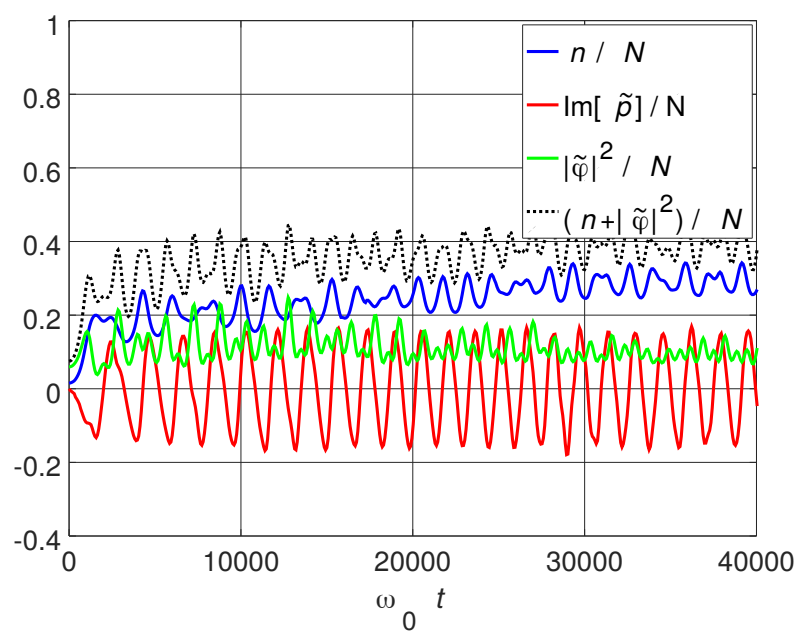

Fig. 4. Magnon density $n(t) / N$ (blue) and imaginary part of the off-diagonal density $\operatorname{Im} \tilde{p}(t) / N$ (red) in the rotating reference frame obtained from the numerical solution of the collisionless kinetic equations 29 - 31 at different levels of approximation discussed in Sect. 3 (a) conventional S-theory, (b) S-theory with mode coupling, (c) S-theory with mode coupling and magnon condensation; the green line represents $\sum_{k}\left|\tilde{\psi}_{\boldsymbol{k}}\right|^{2} / N$. The results are obtained with momentum mesh consisting of $N_{\alpha}=30$ angles and $N_{\varepsilon}=20$ energies as described in the text. The parameters are chosen as $H_{0}=800$ Oe, $H_{1}=50 \mathrm{Oe}, \omega_{0}=13.857 \mathrm{GHz}$, and $d=10 \mu \mathrm{m}$ corresponding to $N=8080$. 
(a)
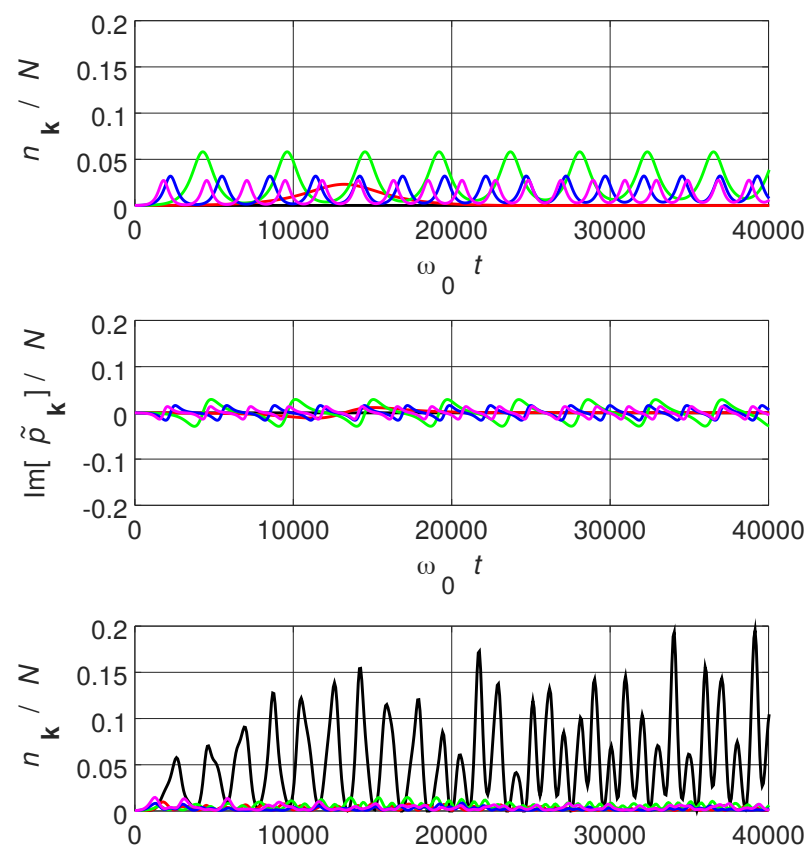

(b)
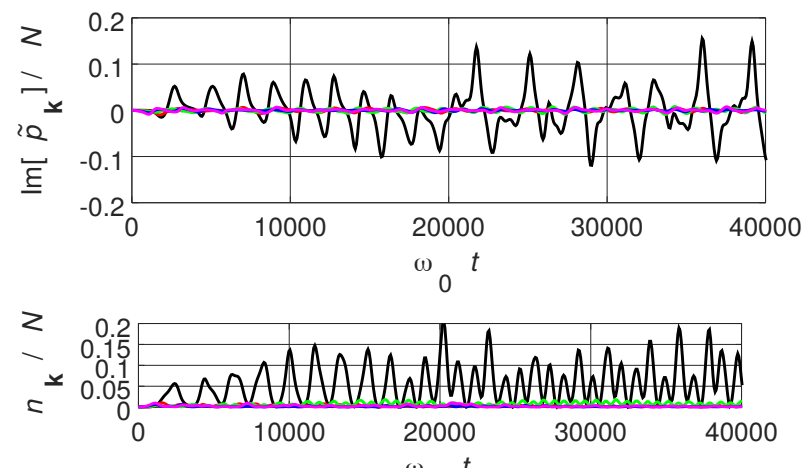

$z \quad \omega_{0}{ }^{t}$

(c)

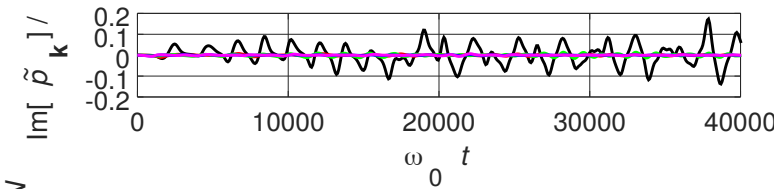

z

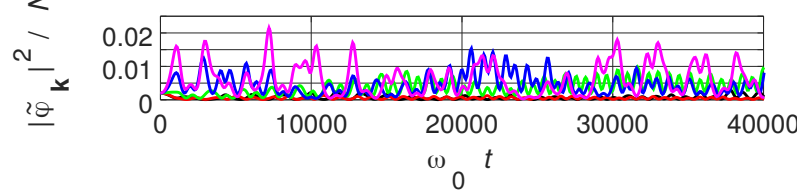

Fig. 5. Time evolution of the diagonal distribution $n_{\boldsymbol{k}}(t) / N$ (top) and the off-diagonal distribution function $\tilde{p}_{\boldsymbol{k}}(t) / N$ (bottom) for different wavevectors satisfying the resonance condition $\varepsilon_{\boldsymbol{k}}=\omega_{0} / 2$. We have chosen six different angles $\theta_{\boldsymbol{k}}$ between 0 and $\pi / 2$ uniformly to be shown. (a) within conventional Stheory, (b) S-theory with mode coupling, (c) S-theory with mode coupling and magnon condensation. The parameters are the same as in Fig. 4

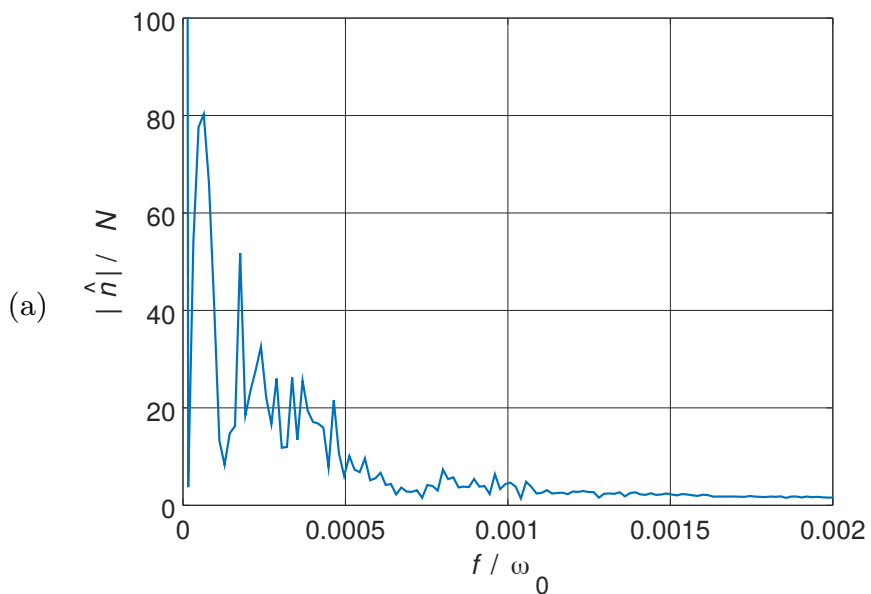

(b)
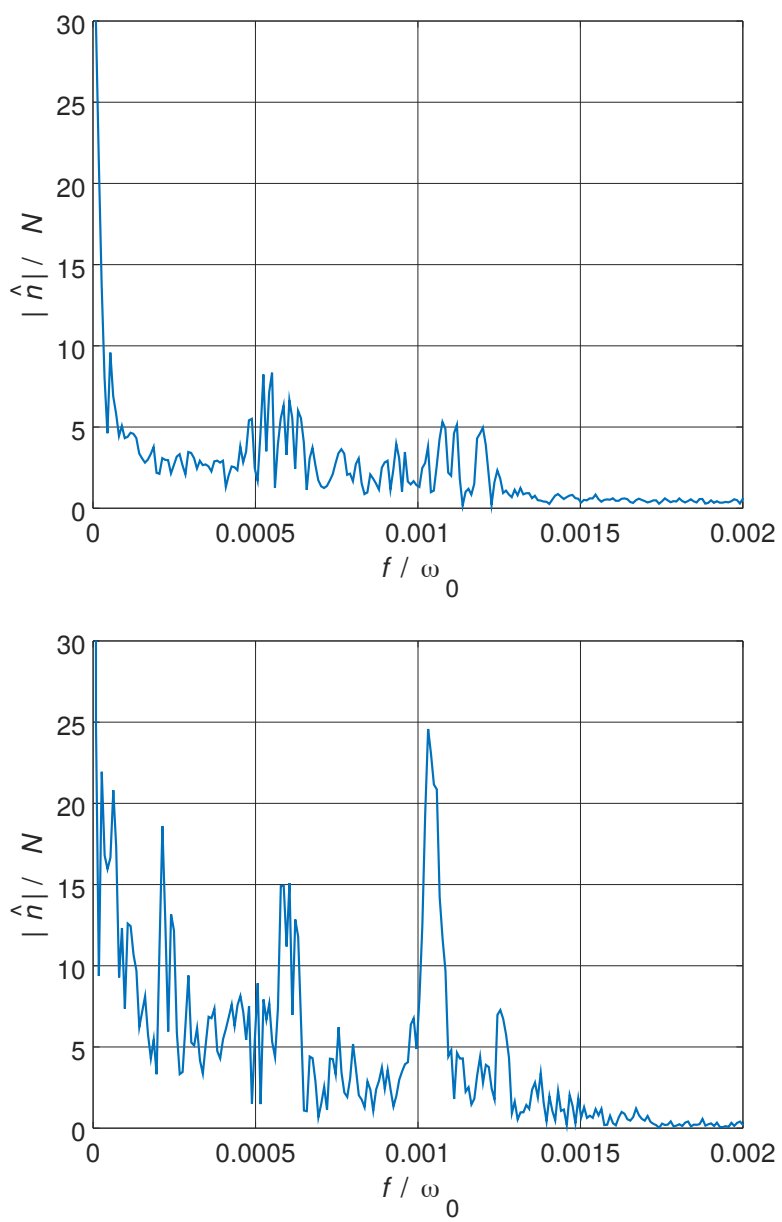

Fig. 6. The absolute value of the discrete Fourier transform $\hat{n}\left(f_{l}\right)=\sum_{j=0}^{N_{t}-1} e^{-2 \pi i f_{l} j} n\left(t_{j}\right)$ of the magnon density $n(t)=$ $\sum_{\boldsymbol{k}} n_{\boldsymbol{k}}(t)$ shown in Fig. 4 , where $f_{l}=\frac{l}{N_{t}}$. (a) Within conventional S-theory, (b) S-theory with mode coupling, (c) S-theory with mode coupling and magnon condensation. In this case the Fourier transform of $n^{c}(t)+|\psi(t)|^{2}$ is shown. 
evolution looks rather different, as shown in Figs. 4 (b) and $5(\mathrm{~b})$. The most striking difference is that now the time-dependence of the magnon density is a superposition of oscillations with different frequencies, while the off-diagonal density $p$ oscillates with fixed frequency and almost constant amplitude. Note also that $n(t) / N$ now oscillates around $n / N \approx 0.30$, which is significantly smaller than the corresponding value $n / N \approx 0.46$ obtained within the mode-decoupling approximation. The time evolution of specific momentum modes shown in Fig. 5(b) exhibits a rather complex behavior which now does not even resemble its mode-decoupled counter-part in Fig. 5 (a). This change is also visible in frequency space (Fig. 6 (b)); the peaks for low frequency become less dominant and higher frequencies emerge.

Finally, let us also take the magnon condensation into account as described in Sect. 3.4. The result of the numerical solution of the coupled integro-differential equations (29)-(32) is shown in Figs. 4 (c) and 5 (c). The time evolution of the total magnon density looks very similar to the corresponding time evolution without magnon condensation, with a slightly smaller time-average $\left(n+|\tilde{\psi}|^{2}\right) / N \approx$ 0.29 . However, the time evolution of representative modes shown in Fig. 5 (c) is again different from the previous two cases. In general, the inclusion of the finite expectation values of the magnon operators leads to faster oscillations involving a broader range of frequencies.

\section{Summary and conclusions}

In this work we have extended S-theory, which is a collisionless kinetic theory for pumped magnon gases, by including the coupling between different modes and the finite expectation values of the magnon operators. The conventional equations of S-theory should then be complemented by an additional equation of motion for the expectation values of the magnon operators which is analogous to the Gross-Pitaevskii equation of a Bose gas.

We have numerically solved collisionless kinetic equations for diagonal- and off-diagonal distribution functions of magnons and have compared the resulting magnon dynamics using three different approximation schemes: conventional S-theory without mode coupling, S-theory with mode coupling, and S-theory with mode coupling and magnon condensation. The time-averaged magnon density in the saturated regime has been found to have the same order of magnitude for all three cases. However, the different approximations lead to a very different time-dependence of the occupation of representative magnon modes with given wavevectors: mode coupling generates a more complex time evolution which still exhibits some periodic structures, which are destroyed if the dynamics of the expectation values of the magnon operators is taken into account.

We have found numerically that a unique solution of the collisionless kinetic equations exists only in some regimes of parameter space and have argued that the solution is unphysical. We conclude that physically meaningful results for the stationary non-equilibrium state of pumped magnon gases can only be obtained if magnon damping is taken into account, either by including the microscopic collision integrals or phenomenologically by adding a damping rate by hand. As discussed in Sect. 4 the latter is usually done in order to justify the modedecoupling approximation within conventional S-theory. While such a procedure can be physically motivated, it is formally not satisfying. We have recently made some progress in this direction [33] by solving the kinetic equations for the magnon distribution in YIG including the microscopic collision integral arising from the cubic magnon vertices.

\section{Author contribution statement}

Viktor Hahn carried out all numerical and analytical calculations; Peter Kopietz has checked most of the analytical calculations. Both authors have discussed the results and have contributed to writing the paper.

\section{APPENDIX: HAMILTONIAN FOR PUMPED MAGNONS IN YIG}

To make this work self-contained, we outline here the main technical steps in the derivation of the magnon Hamiltonian given in Eqs. (9) and (15) from the effective spin Hamiltonian (6), see also Refs. [34,37. As usual, we express the components of the spin operators $\boldsymbol{S}_{i}$ in terms of boson annihilation and creation operators $b_{i}$ and $b_{i}^{\dagger}$ using the Holstein-Primakoff transformation [30] and expand the effective boson Hamiltonian in powers of $1 / S$, see Eq. (8). Transforming to momentum space,

$$
b_{i}=\frac{1}{\sqrt{N}} \sum_{\boldsymbol{k}} \mathrm{e}^{i \boldsymbol{k} \cdot \boldsymbol{r}_{i}} b_{\boldsymbol{k}}
$$

the quadratic part $\mathcal{H}_{2}(t)$ of the effective boson Hamiltonian can be written as 34

$$
\begin{aligned}
\mathcal{H}_{2}(t)= & \sum_{\boldsymbol{k}}\left[A_{\boldsymbol{k}} b_{\boldsymbol{k}}^{\dagger} b_{\boldsymbol{k}}+\frac{B_{\boldsymbol{k}}}{2}\left(b_{\boldsymbol{k}}^{\dagger} b_{-\boldsymbol{k}}^{\dagger}+b_{-\boldsymbol{k}} b_{\boldsymbol{k}}\right)\right] \\
& +h_{1} \cos \left(\omega_{0} t\right) \sum_{\boldsymbol{k}} b_{\boldsymbol{k}}^{\dagger} b_{\boldsymbol{k}},
\end{aligned}
$$

where

$$
\begin{aligned}
& A_{\boldsymbol{k}}=h_{0}+S\left(J_{0}-J_{\boldsymbol{k}}\right)+S\left[D_{\mathbf{0}}^{z z}-\frac{1}{2}\left(D_{\boldsymbol{k}}^{x x}+D_{\boldsymbol{k}}^{y y}\right)\right] \\
& B_{\boldsymbol{k}}=-\frac{S}{2}\left[D_{\boldsymbol{k}}^{x x}-2 i D_{\boldsymbol{k}}^{x y}-D_{\boldsymbol{k}}^{y y}\right],
\end{aligned}
$$

and $J_{k}$ and $D_{k}^{\alpha \beta}$ are the Fourier transforms of the exchange and dipolar couplings,

$$
\begin{aligned}
J_{\boldsymbol{k}} & =\sum_{i} \mathrm{e}^{-i \boldsymbol{k} \cdot \boldsymbol{r}_{i j}} J_{i j}, \\
D_{\boldsymbol{k}}^{\alpha \beta} & =\sum_{i} \mathrm{e}^{-i \boldsymbol{k} \cdot \boldsymbol{r}_{i j}} D_{i j}^{\alpha \beta} .
\end{aligned}
$$


The cubic and quartic parts of the Hamiltonian read 34

$$
\begin{aligned}
\mathcal{H}_{3}= & \frac{1}{\sqrt{N}} \sum_{\boldsymbol{k}_{1}, \boldsymbol{k}_{2}, \boldsymbol{k}_{3}} \delta_{\boldsymbol{k}_{1}+\boldsymbol{k}_{2}+\boldsymbol{k}_{3}, 0} \frac{1}{2 !}\left[\Gamma_{1 ; 2,3}^{\bar{b} b b} b_{-1}^{\dagger} b_{2} b_{3}\right. \\
& \left.+\Gamma_{1,2 ; 3}^{\bar{b} \bar{b} b} b_{-1}^{\dagger} b_{-2}^{\dagger} b_{3}\right], \\
\mathcal{H}_{4}= & \frac{1}{N} \sum_{\boldsymbol{k}_{1}, \ldots, \boldsymbol{k}_{4}} \delta_{\boldsymbol{k}_{1}+\boldsymbol{k}_{2}+\boldsymbol{k}_{3}+\boldsymbol{k}_{4}, 0}\left[\frac{1}{(2 !)^{2}} \Gamma_{1,2 ; 3,4}^{\bar{b} \bar{b} b b} b_{-1}^{\dagger} b_{-2}^{\dagger} b_{3} b_{4}\right. \\
& \left.+\frac{1}{3 !} \Gamma_{1 ; 2,3,4}^{\bar{b} b b b} b_{-1}^{\dagger} b_{2} b_{3} b_{4}+\frac{1}{3 !} \Gamma_{1,2,3 ; 4}^{\bar{b} \bar{b} \bar{b} b} b_{-1}^{\dagger} b_{-2}^{\dagger} b_{-3}^{\dagger} b_{4}\right],
\end{aligned}
$$

where the cubic and quartic vertices are given by

$$
\begin{aligned}
& \Gamma_{1 ; 2,3}^{\bar{b} b b}=\sqrt{\frac{S}{2}}\left[D_{\boldsymbol{k}_{2}}^{z y}-i D_{\boldsymbol{k}_{2}}^{z x}+D_{\boldsymbol{k}_{3}}^{z y}-i D_{\boldsymbol{k}_{3}}^{z x}\right. \\
& \left.+\frac{1}{2}\left(D_{\mathbf{0}}^{z y}-i D_{\mathbf{0}}^{z x}\right)\right] \text {, } \\
& \Gamma_{1,2 ; 3}^{\bar{b} \bar{b} b}=\left(\Gamma_{3 ; 2,1}^{\bar{b} b b}\right)^{*} \\
& \Gamma_{1,2 ; 3,4}^{\bar{b} \bar{b} b b}=-\frac{1}{2}\left[J_{\boldsymbol{k}_{1}+\boldsymbol{k}_{3}}+J_{\boldsymbol{k}_{2}+\boldsymbol{k}_{3}}+J_{\boldsymbol{k}_{1}+\boldsymbol{k}_{4}}+J_{\boldsymbol{k}_{2}+\boldsymbol{k}_{4}}\right. \\
& +D_{\boldsymbol{k}_{1}+\boldsymbol{k}_{3}}^{z z}+D_{\boldsymbol{k}_{2}+\boldsymbol{k}_{3}}^{z z}+D_{\boldsymbol{k}_{1}+\boldsymbol{k}_{4}}^{z z}+D_{\boldsymbol{k}_{2}+\boldsymbol{k}_{4}}^{z z} \\
& \left.-\sum_{i=1}^{4}\left(J_{\boldsymbol{k}_{i}}-2 D_{\boldsymbol{k}_{i}}^{z z}\right)\right] \text {, } \\
& \Gamma_{1 ; 2,3,4}^{\bar{b} b b b}=\frac{1}{4}\left[D_{\boldsymbol{k}_{2}}^{x x}-2 i D_{\boldsymbol{k}_{2}}^{x y}-D_{\boldsymbol{k}_{2}}^{y y}+D_{\boldsymbol{k}_{3}}^{x x}-2 i D_{\boldsymbol{k}_{3}}^{x y}-D_{\boldsymbol{k}_{3}}^{y y}\right. \\
& \left.+D_{\boldsymbol{k}_{4}}^{x x}-2 i D_{\boldsymbol{k}_{4}}^{x y}-D_{\boldsymbol{k}_{4}}^{y y}\right] \text {, } \\
& \Gamma_{1,2,3 ; 4}^{\bar{b} \bar{b} \bar{b} b}=\left(\Gamma_{4 ; 1,2,3}^{\bar{b} b b b}\right)^{*} \text {. }
\end{aligned}
$$

To diagonalize the time-independent part of $\mathcal{H}_{2}(t)$ we use a canonical transformation

$$
\left(\begin{array}{c}
b_{k} \\
b_{-k}^{\dagger}
\end{array}\right)=\left(\begin{array}{cc}
u_{k} & -v_{k} \\
-v_{\mathbf{k}}^{*} & u_{k}
\end{array}\right)\left(\begin{array}{c}
a_{k} \\
a_{-k}^{\dagger}
\end{array}\right)
$$

where

$$
\begin{aligned}
& u_{k}=\sqrt{\frac{A_{k}+\varepsilon_{k}}{2 \varepsilon_{k}}}, \\
& v_{k}=\frac{B_{k}}{\left|B_{k}\right|} \sqrt{\frac{A_{k}-\varepsilon_{k}}{2 \varepsilon_{k}}}
\end{aligned}
$$

and the magnon dispersion is given by

$$
\varepsilon_{\boldsymbol{k}}=\sqrt{A_{\boldsymbol{k}}^{2}-\left|B_{\boldsymbol{k}}\right|^{2}}
$$

Due to the time dependence of the last term in Eq. A2 , the quadratic part of the Hamiltonian has also off-diagonal terms,

$$
\begin{aligned}
\mathcal{H}_{2}(t) & =\sum_{\boldsymbol{k}}\left[\varepsilon_{\boldsymbol{k}} a_{\boldsymbol{k}}^{\dagger} a_{\boldsymbol{k}}+\frac{\varepsilon_{\boldsymbol{k}}-A_{\boldsymbol{k}}}{2}\right. \\
& \left.+h_{1} \cos \left(\omega_{0} t\right)\left(\frac{A_{\boldsymbol{k}}}{\varepsilon_{\boldsymbol{k}}} a_{\boldsymbol{k}}^{\dagger} a_{\boldsymbol{k}}-\frac{\varepsilon_{\boldsymbol{k}}-A_{\boldsymbol{k}}}{2 \varepsilon_{\boldsymbol{k}}}\right)\right] \\
& +\sum_{\boldsymbol{k}}\left[V_{\boldsymbol{k}} \cos \left(\omega_{0} t\right) a_{\boldsymbol{k}}^{\dagger} a_{-\boldsymbol{k}}^{\dagger}+V_{\boldsymbol{k}}^{*} \cos \left(\omega_{0} t\right) a_{-\boldsymbol{k}} a_{\boldsymbol{k}}\right]
\end{aligned}
$$

where

$$
V_{\boldsymbol{k}}=-\frac{h_{1} B_{\boldsymbol{k}}}{2 \varepsilon_{\boldsymbol{k}}}
$$

At this point we transform to the rotating reference frame using another canonical transformation given in Eq. (13). In rotating-wave approximation all terms which still exhibit an explicit time-dependence in the rotating reference frame are neglected so that we arrive at the timedependent quadratic Hamiltonian in Eq. (14).

In terms of the magnon operators $\tilde{a}_{\boldsymbol{k}}$ and $\tilde{a}_{\boldsymbol{k}}^{\dagger}$ in the rotating reference frame the cubic and quartic parts of the Hamiltonian, $\tilde{\mathcal{H}}_{3}$ and $\tilde{\mathcal{H}}_{4}$, are 34 ]

$$
\begin{aligned}
\tilde{\mathcal{H}}_{3}= & \frac{1}{\sqrt{N}} \sum_{\boldsymbol{k}_{1}, \boldsymbol{k}_{2}, \boldsymbol{k}_{3}} \delta_{\boldsymbol{k}_{1}+\boldsymbol{k}_{2}+\boldsymbol{k}_{3}, 0}\left[\frac{1}{2} \Gamma_{1 ; 2,3}^{\bar{a} a a} e^{-i \omega_{0} t / 2} \tilde{a}_{-1}^{\dagger} \tilde{a}_{2} \tilde{a}_{3}\right. \\
& +\frac{1}{2} \Gamma_{1,2 ; 3}^{\bar{a} \bar{a} a} e^{i \omega_{0} t / 2} \tilde{a}_{-1}^{\dagger} \tilde{a}_{-2}^{\dagger} \tilde{a}_{3} \\
& +\frac{1}{3 !} \Gamma_{1,2,3}^{a a a} e^{-3 i \omega_{0} t / 2} \tilde{a}_{1} \tilde{a}_{2} \tilde{a}_{3} \\
& \left.+\frac{1}{3 !} \Gamma_{1,2,3}^{\bar{a} \bar{a} \bar{a}} e^{3 i \omega_{0} t / 2} \tilde{a}_{-1}^{\dagger} \tilde{a}_{-2}^{\dagger} \tilde{a}_{-3}^{\dagger}\right] \\
\tilde{\mathcal{H}}_{4}= & \frac{1}{N} \sum_{\boldsymbol{k}_{1}, \ldots, \boldsymbol{k}_{4}} \delta_{\boldsymbol{k}_{1}+\cdots+\boldsymbol{k}_{4}, 0}\left[\frac{1}{(2 !)^{2}} \Gamma_{1,2 ; 3,4}^{\bar{a} \bar{a} a a} \tilde{a}_{-1}^{\dagger} \tilde{a}_{-2}^{\dagger} \tilde{a}_{3} \tilde{a}_{4}\right. \\
& +\frac{1}{3 !} \Gamma_{1 ; 2,3,4}^{\bar{a} a a a} e^{-i \omega_{0} t} \tilde{a}_{-1}^{\dagger} \tilde{a}_{2} \tilde{a}_{3} \tilde{a}_{4} \\
& +\frac{1}{3 !} \Gamma_{1,2,3 ; 4}^{\bar{a} \bar{a} \bar{a} a} e^{i \omega_{0} t} \tilde{a}_{-1}^{\dagger} \tilde{a}_{-2}^{\dagger} \tilde{a}_{-3}^{\dagger} \tilde{a}_{4} \\
& +\frac{1}{4 !} \Gamma_{1,2,3,4}^{a a a a} e^{-2 i \omega_{0} t} \tilde{a}_{1} \tilde{a}_{2} \tilde{a}_{3} \tilde{a}_{4} \\
& \left.+\frac{1}{4 !} \Gamma_{1,2,3,4}^{\bar{a} \bar{a} \bar{a} \bar{a}} e^{2 i \omega_{0} t} \tilde{a}_{-1}^{\dagger} \tilde{a}_{-2}^{\dagger} \tilde{a}_{-3}^{\dagger} \tilde{a}_{-4}^{\dagger}\right]
\end{aligned}
$$

with cubic vertices

$$
\begin{aligned}
\Gamma_{1,2,3}^{a a a}= & -\Gamma_{1 ; 2,3}^{\bar{b} b} v_{1} u_{2} u_{3}-\Gamma_{2 ; 1,3}^{\bar{b} b b} v_{2} u_{1} u_{3}-\Gamma_{3 ; 1,2}^{\bar{b} b b} v_{3} u_{1} u_{3} \\
& +\Gamma_{1,2 ; 3}^{\bar{b} \bar{b} b} v_{1} v_{2} u_{3}+\Gamma_{2,3 ; 1}^{\bar{b} \bar{b} b} v_{2} v_{3} u_{1}+\Gamma_{1,3 ; 2}^{\bar{b} \bar{b} b} v_{1} v_{3} u_{2}
\end{aligned}
$$

$$
\begin{aligned}
\Gamma_{1 ; 2,3}^{\bar{a} a a}= & \Gamma_{1 ; 2,3}^{\bar{b} b b} u_{1} u_{2} u_{3}+\Gamma_{2 ; 1,3}^{\bar{b} b b} v_{1} v_{2} u_{3}+\Gamma_{3 ; 1,2}^{\bar{b} b b} v_{1} v_{3} u_{2} \\
& -\Gamma_{3,2 ; 1}^{\bar{b} \bar{b} b} v_{3} v_{2} v_{1}-\Gamma_{1,2 ; 3}^{\bar{b} \bar{b} b} v_{2} u_{1} u_{3}-\Gamma_{1,3 ; 2}^{\bar{b} \bar{b} b} v_{3} u_{1} u_{2}
\end{aligned}
$$

$$
\begin{aligned}
& \Gamma_{1,2 ; 3}^{\bar{a} \bar{a} a}=\left(\Gamma_{3 ; 2,1}^{\bar{a} a a}\right)^{*}, \\
& \Gamma_{1,2,3}^{\bar{a} \bar{a} \bar{a}}=\left(\Gamma_{1,2,3}^{a a a}\right)^{*},
\end{aligned}
$$


and quartic vertices

$$
\begin{aligned}
\Gamma_{1,2,3,4}^{a a a a}= & \Gamma_{1,2 ; 3,4}^{\bar{b} \bar{b} b b} u_{1} u_{2} v_{3} v_{4}+\Gamma_{1,3 ; 2,4}^{\bar{b} \bar{b} b b} u_{1} u_{3} v_{2} v_{4} \\
& +\Gamma_{1,4 ; 2,3}^{\bar{b} \bar{b} b b} u_{1} u_{4} v_{2} v_{3}+\Gamma_{2,3 ; 1,4}^{\bar{b} \bar{b} b b} u_{2} u_{3} v_{1} v_{4} \\
& +\Gamma_{2,4 ; 1,3}^{\bar{b} \bar{b} b b} u_{2} u_{4} v_{1} v_{3}+\Gamma_{3,4 ; 1,2}^{\bar{b} \bar{b} b b} u_{3} u_{4} v_{1} v_{2} \\
& -\Gamma_{4 ; 1,2,3}^{\bar{b} b b b} u_{1} u_{2} u_{3} v_{4}-\Gamma_{3 ; 1,2,4}^{\bar{b} b b b} u_{1} u_{2} u_{4} v_{3} \\
& -\Gamma_{2 ; 1,3,4}^{\bar{b} b b b} u_{1} u_{3} u_{4} v_{2}-\Gamma_{1 ; 2,3,4}^{\bar{b} b b b} u_{2} u_{3} u_{4} v_{1} \\
& -\Gamma_{2,3,4 ; 1}^{\bar{b} \bar{b} \bar{b} b} u_{1} v_{2} v_{3} v_{4}-\Gamma_{1,3,4 ; 2}^{\bar{b} \bar{b} \bar{b} b} u_{2} v_{1} v_{3} v_{4} \\
& -\Gamma_{1,2,4 ; 3}^{\bar{b} \bar{b} b} u_{3} v_{1} v_{2} v_{4}-\Gamma_{1,2,3 ; 4}^{\bar{b} \bar{b} \bar{b} b} u_{4} v_{1} v_{2} v_{3}
\end{aligned}
$$

(A18a)

$$
\begin{aligned}
\Gamma_{1 ; 2,3,4}^{\bar{a} a a a}= & -\Gamma_{2,1 ; 3,4}^{\bar{b} \bar{b} b b} u_{2} v_{1} v_{3} v_{4}-\Gamma_{3,1 ; 2,4}^{\bar{b} \bar{b} b b} u_{3} v_{1} v_{2} v_{4} \\
& -\Gamma_{4,1 ; 2,3}^{\bar{b} \bar{b} b b} u_{4} v_{1} v_{2} v_{3}-\Gamma_{2,3 ; 1,4}^{\bar{b} \bar{b} b b} u_{2} u_{3} u_{1} v_{4} \\
& -\Gamma_{2,4 ; 1,3}^{\bar{b} \bar{b} b b} u_{2} u_{4} u_{1} v_{3}-\Gamma_{3,4 ; 1,2}^{\bar{b} \bar{b} b b} u_{3} u_{4} u_{1} v_{2} \\
& +\Gamma_{1 ; 2,3,4}^{\bar{b} b b b} u_{1} u_{2} u_{3} u_{4}+\Gamma_{4 ; 3,2,1}^{\bar{b} b b b} u_{3} u_{2} v_{1} v_{4} \\
& +\Gamma_{3 ; 4,2,1}^{\bar{b} b b b} u_{4} u_{2} v_{1} v_{3}+\Gamma_{2 ; 4,3,1}^{\bar{b} b b b} u_{4} u_{3} v_{1} v_{2} \\
& +\Gamma_{1,2,3 ; 4}^{\bar{b} \bar{b} \bar{b} b} u_{4} u_{1} v_{2} v_{3}+\Gamma_{1,2,4 ; 3}^{\bar{b} \bar{b} b} u_{3} u_{1} v_{2} v_{4} \\
& +\Gamma_{1,3,4 ; 2}^{\bar{b} \bar{b} \bar{b} b} u_{2} u_{1} v_{3} v_{4}+\Gamma_{4,3,2 ; 1}^{\bar{b} \bar{b} b} v_{4} v_{2} v_{3} v_{1}
\end{aligned}
$$

$$
\begin{aligned}
\Gamma_{1,2 ; 3,4}^{\bar{a} \bar{a} a a}= & \Gamma_{1,2 ; 3,4}^{\bar{b} \bar{b} b b} u_{1} u_{2} u_{3} u_{4}+\Gamma_{1,3 ; 4,2}^{\bar{b} \bar{b} b b} u_{1} u_{4} v_{3} v_{2} \\
& +\Gamma_{1,4 ; 3,2}^{\bar{b} \bar{b} b b} u_{1} u_{3} v_{4} v_{2}+\Gamma_{2,3 ; 4,1}^{\bar{b} \bar{b} b b} u_{2} u_{4} v_{3} v_{1} \\
& +\Gamma_{2,4 ; 3,1}^{\bar{b} \bar{b} b b} u_{2} u_{3} v_{4} v_{1}+\Gamma_{3,4 ; 2,1}^{\bar{b} \bar{b} b b} v_{1} v_{2} v_{3} v_{4} \\
& -\Gamma_{4 ; 3,2,1}^{\bar{b} b b b} u_{3} v_{2} v_{1} v_{4}-\Gamma_{3 ; 4,2,1}^{\bar{b} b b b} u_{4} v_{2} v_{1} v_{3} \\
& -\Gamma_{2 ; 3,4,1}^{\bar{b} b b b} u_{2} u_{3} u_{4} v_{1}-\Gamma_{1 ; 3,4,2}^{\bar{b} b b b} u_{1} u_{3} u_{4} v_{2} \\
& -\Gamma_{2,3,4 ; 1}^{\bar{b} \bar{b} \bar{b} b} u_{2} v_{3} v_{4} v_{1}-\Gamma_{1,3,4 ; 2}^{\bar{b} \bar{b} \bar{b} b} u_{1} v_{3} v_{4} v_{2} \\
& -\Gamma_{1,2,4 ; 3}^{\bar{b} \bar{b} \bar{b} b} u_{1} u_{2} u_{3} v_{4}-\Gamma_{1,2,3 ; 4}^{\bar{b} \bar{b} \bar{b} b} u_{1} u_{2} u_{4} v_{3}
\end{aligned}
$$

$$
\begin{aligned}
\Gamma_{1,2,3,4}^{\bar{a} \bar{a} \bar{a} \bar{a}} & =\Gamma_{1,2,3,4}^{a a a a}, \\
\Gamma_{1,2,3 ; 4}^{\bar{a} \bar{a} \bar{a} a} & =\left(\Gamma_{4 ; 3,2,1}^{\bar{a} a a a}\right)^{*} .
\end{aligned}
$$

Finally, let us specify the coefficients $A_{\boldsymbol{k}}, B_{\boldsymbol{k}}$, and $D_{\boldsymbol{k}}^{\alpha \beta}$ for a thin film of YIG. We assume that the thickness $d$ in $x$-direction is small compared to the extensions in $y$ - and $z$-direction. We are only interested in the dispersion of the lowest magnon band and therefore use an effective in-plane Hamiltonian to derive the dispersion of the lowest magnon band. We use the uniform mode approximation ignoring the fact that the system is not translationally invariant in the $x$-direction and replace the transverse mode by plane waves. This is valid for periodic boundary conditions in all directions 37. The coefficients $A_{\boldsymbol{k}}$ and $B_{\boldsymbol{k}}$ defined in Eqs. A3 and (A4) can then be written as

$$
\begin{aligned}
A_{\boldsymbol{k}}= & h_{0}+J S\left[4-2 \cos \left(k_{y} a\right)-2 \cos \left(k_{z} a\right)\right] \\
& -\frac{S}{2}\left(D_{\boldsymbol{k}}^{x x}+D_{\boldsymbol{k}}^{y y}\right)+\frac{\Delta}{3} \\
B_{\boldsymbol{k}}= & -\frac{S}{2}\left(D_{\boldsymbol{k}}^{x x}-D_{\boldsymbol{k}}^{y y}\right)
\end{aligned}
$$

where the Fourier transforms of the dipole matrix elements are 37 .

$$
\begin{aligned}
& D_{\boldsymbol{k}}^{x x}=\frac{4 \pi \mu^{2}}{a^{3}}\left[\frac{1}{3}-f_{\boldsymbol{k}}\right], \\
& D_{\boldsymbol{k}}^{y y}=\frac{4 \pi \mu^{2}}{a^{3}}\left[\frac{1}{3}-\left(1-f_{\boldsymbol{k}}\right) \sin ^{2} \theta_{\boldsymbol{k}}\right], \\
& D_{\boldsymbol{k}}^{z z}=\frac{4 \pi \mu^{2}}{a^{3}}\left[\frac{1}{3}-\left(1-f_{\boldsymbol{k}}\right) \cos ^{2} \theta_{\boldsymbol{k}}\right], \\
& D_{\boldsymbol{k}}^{x y}=D_{\boldsymbol{k}}^{y x}=0,
\end{aligned}
$$

with the form factor given in Eq. (11). Note that for $\boldsymbol{k}=0$ there is the general relation [41,42, 43, 44]

$$
D_{\boldsymbol{k}=0}^{\alpha \beta}=\frac{4 \pi \mu^{2}}{a^{3}}\left[\frac{1}{3}-\mathcal{N}_{\alpha}\right] \delta_{\alpha \beta},
$$

where $\mathcal{N}_{\alpha}$ is the geometry-dependent demagnetization factor. Eqs. A21a - A21c are a special case of this relation.

\section{References}

1. H. Suhl, The theory of ferromagnetic resonance at high signal powers, J. Phys. Chem. Solids 1, 209 (1957).

2. E. Schlömann, J. J. Green, and U. Milano, Recent Developments in Ferromagnetic Resonance at High Power Levels, J. Appl. Phys. 31, 386S (1960); E. Schlömann and R. I. Joseph, Instability of Spin Waves and Magnetostatic Modes in a Microwave Magnetic Field Applied Parallel to the dc Field, ibid. 32, 1006 (1961); E. Schlömann and J. J. Green, Spin-Wave Growth Under Parallel Pumping, ibid. 34, 1291 (1963).

3. V. E. Zakharov, V. S. L'vov, and S. S. Starobinets, Stationary nonlinear theory of parametric excitation of waves, Zh. Eksp. Teor. Fiz. 59, 1200 (1970) [Sov. Phys. JETP 32, 656 (1971)].

4. V. E. Zakharov, V. S. L'vov, and S. S. Starobinets, Spinwave turbulence beyond the parametric excitation threshold, Usp. Fiz. Nauk 114, 609 (1974) [Sov. Phys.-Usp. 17, $896(1975)]$.

5. V. Charepanov, I. Kolokolov, and V. S. L'vov, The saga of YIG: spectra, thermodynamics, interaction and relaxation of magnons in a complex magnet, Phys. Rept. 229, 81 (1993).

6. C. B. Araujo, Quantum-statistical theory of the nonlinear excitation of magnons in parallel pumping experiments, Phys. Rev. B 10, 3961 (1974).

7. V. M. Tsukernik and R. P. Yankelevich, Stationary distribution of magnons following parametric excitation in ferromagnetic substance, Zh. Eksp. Teor. Fiz. 68, 2116 (1975) [Sov. Phys. JETP 41, 1059 (1976)].

8. I. A. Vinikovetskii, A. M. Frishman, and V. M. Tsukernik, Kinetic equation for a system of parametrically excited spin waves, Zh. Eksp. Teor. Fiz. 76, 2110 (1979) [Sov. Phys. JETP 49, 1067 (1979)].

9. A. V. Lavrinenko, V. S. L'vov, G. A. Melkov, and V. B. Cherepanov, "Kinetic" instability of a strongly nonequilibrium system of spin waves and tunable radiation of a ferrite, Zh. Eksp. Teor. Fiz. 81, 1022 (1981) [Sov. Phys. JETP 54, 542 (1981)]. 
10. A. A. Zvyagin, V. Ya. Serebryannyi, A. M. Frishman, and V. M. Tsukernik, Dynamics of spin waves under parametric excitation by a stepped periodic field of arbitrary amplitude, Fiz. Nizk. Temp. 8, 1205 (1982) [Sov. J. Low Temp. Phys. 8, 612 (1982)].

11. A. A. Zvyagin and V. M. Tsukernik, A change in equilibrium configuration of magnetic system during parametric excitation, Fiz. Nizk. Temp. 11, 88 (1985) [Sov. J. Low Temp. Phys. 11, 47 (1985)].

12. S. P. Lim and D. L. Huber, Microscopic theory of spinwave instabilities in parallel-pumped easy-plane ferromagnets, Phys. Rev. B 37, 5426 (1988); Possible mechanism for limiting the number of modes in spin-wave instabilities in parallel pumping, ibid. 41, 9283 (1990).

13. Yu. D. Kalafati and V. L. Safonov, Thermodynamic approach in the theory of paramagnetic resonance of magnons, Zh. Eksp. Teor. Fiz. 95, 2009 (1989) [Sov. Phys. JETP 68, 1162 (1989)]

14. V. S. L'vov, Wave Turbulence Under Parametric Excitations, (Springer, Berlin, 1994).

15. A. A. Zvyagin, Re-distribution (condensation) of magnons in a ferromagnet under pumping, Fiz. Nizk. Temp. 33, 1248 (2007) [Sov. J. Low Temp. Phys. 33, 948 (2007)].

16. S. M. Rezende, Theory of microwave superradiance from a Bose-Einstein condensate of magnons, Phys. Rev. B 79, 060410(R) (2009); Theory of coherence in Bose-Einstein condensation phenomena in a microwave-driven interacting magnon gas, ibid., 174411 (2009).

17. T. Kloss, A. Kreisel, and P. Kopietz, Parametric pumping and kinetics of magnons in dipolar ferromagnets, Phys. Rev. B 81, 104308 (2010).

18. V. L. Safonov, Nonequilibrium Magnons, (Wiley-VCH, Weinheim, Germany, 2013).

19. D. V. Slobodianiuk and O. V. Prokopenko, Kinetics of Strongly Nonequilibrium Magnon Gas Leading to BoseEinstein Condensation, J. Nano- Electron. Phys. 9, 03033 (2017).

20. S. O. Demokritov, V. E. Demidov, O. Dzyapko, G. A. Melkov, A. A. Serga, B. Hillebrands, and A. N. Slavin, Bose-Einstein condensation of quasi-equilibrium magnons at room temperature under pumping, Nature 443, 430 (2006).

21. V. E. Demidov, O. Dzyapko, S. O. Demokritov, G. A. Melkov, and A. N. Slavin, Thermalization of a Parametrically Driven Magnon Gas Leading to Bose-Einstein Condensation, Phys. Rev. Lett. 99, 037205 (2007).

22. O. Dzyapko, V. E. Demidov, S. O. Demokritov, G. A. Melkov, and A. N. Slavin, Direct observation of BoseEinstein condensation in a parametrically driven gas of magnons, New J. Phys. 9, 64 (2007).

23. V. E. Demidov, O. Dzyapko, S. O. Demokritov, G. A. Melkov, and A. N. Slavin, Observation of Spontaneous Coherence in Bose-Einstein Condensate of Magnons, Phys. Rev. Lett. 100, 047205 (2008).

24. S. O. Demokritov, V. E. Demidov, O. Dzyapko, G. A. Melkov, and A. N. Slavin, Quantum coherence due to Bose-Einstein condensation of parametrically driven magnons, New J. Phys. 10, 045029 (2008).

25. V. E. Demidov, O. Dzyapko, M. Buchmeier, T. Stockhoff, G. Schmitz, G. A. Melkov, and S. O. Demokritov, Magnon Kinetics and Bose-Einstein Condensation Studied in Phase Space, Phys. Rev. Lett. 101, 257201 (2008).
26. A. A. Serga, V. S. Tiberkevich, C. W. Sandweg, V. I. Vasyuchka, D. A. Bozhko, A. V. Chumak, T. Neumann, B. Obry, G. A. Melkov, A. N. Slavin, and B. Hillebrands, Bose-Einstein condensation in an ultra-hot gas of pumped magnons, Nat. Comm. 5, 3452 (2014).

27. P. Clausen, D. A. Bozhko, V. I. Vasyuchka, B. Hillebrands, G. A. Melkov, and A. A. Serga, Stimulated thermalization of a parametrically driven magnon gas as a prerequisite for Bose-Einstein magnon condensation, Phys. Rev. B 91, 220402(R) (2015).

28. P. Clausen, D. A. Bozhko, V. I. Vasyuchka, G. A. Melkov, B. Hillebrands, and A. A. Serga, Supercurrent in a roomtemperature Bose-Einstein magnon condensate, Nature Physics 12, 1057 (2016).

29. A. Rückriegel and P. Kopietz, Rayleigh-Jeans Condensation of Pumped Magnons in Thin-Film Ferromagnets, Phys. Rev. Lett. 115, 157203 (2015).

30. T. Holstein and H. Primakoff, Field Dependence of the Intrinsic Domain Magnetization of a Ferromagnet, Phys. Rev. 58, 1098 (1940).

31. By quantizing the spin operators in a proper timedependent reference frame, the magnon operators can always be defined such that the expectation values $\left\langle a_{\boldsymbol{k}}(t)\right\rangle$ vanish identically, see A. Rückriegel, A. Kreisel, and P. Kopietz, Time-dependent spin-wave theory, Phys. Rev. B $\mathbf{8 5}, 054422(2012)$

32. J. Fricke, Transportgleichungen für quantenmechanische Vielteilchensysteme, (Cuvillier-Verlag, Göttingen, 1996).

33. Very recently we have succeeded to solve the quantum kinetic equations for the magnon distribution in YIG with a microscopically derived collision integral to explain the effect of confluent magnon damping on the parametric resonance of magnons in YIG reported by T. B. Noack, V. I. Vasyuchka, D. A. Bozhko, B. Heinz, P. Frey, D. V. Slobodianiuk, O. V. Prokopenko, G. A. Melkov, P. Kopietz, B. Hillebrands, and A. A. Serga, Enhancement of the Spin Pumping Effect by Magnon Confluence Process in YIG/Pt Bilayers, Phys. Status Solidi B, 256, 1900121 (2019).

34. J. Hick, F. Sauli, A. Kreisel, and P. Kopietz, Bose-Einstein condensation at finite momentum and magnon condensation in thin film ferromagnets, Eur. Phys. J. B 78, 429 (2010).

35. A. Rückriegel, P. Kopietz, D. A. Bozhko, A. A. Serga, and B. Hillebrands, Magnetoelastic modes and lifetime of magnons in thin yttrium iron garnet films, Phys. Rev. B 89, 184413 (2014).

36. S. M. Rezende, F. M. de Aguiar, and A. Azevedo, Magnon excitation by spin-polarized direct currents in magnetic nanostructures, Phys. Rev. B 73, 094402 (2006).

37. A. Kreisel, F. Sauli, L. Bartosch, and P. Kopietz, Microscopic spin-wave theory for yttrium-iron garnet films, Eur. Phys. J. B 71, 59 (2009).

38. I. S. Tupitsyn, P. C. E. Stamp, and A. L. Burin, Stability of Bose-Einstein Condensates of Hot Magnons in Yttrium Iron Garnet Films, Phys. Rev. Lett. 100, 257202 (2008).

39. B. A. Kalinikos, and A. N. Slavin, Theory of dipoleexchange spin wave spectrum for ferromagnetic films with mixed exchange boundary conditions, J. Phys. C 19, 7013 (1986).

40. R. N. Costa Filho, M.G. Cottam, and G.A. Farias, Microscopic theory of dipole-exchange spin waves in ferromagnetic films: Linear and nonlinear processes, Phys. Rev. B 62, 6545 (2000). 
41. M. H. Cohen and F. Keffer, Dipolar Sums in the Primitive Cubic Lattices, Phys. Rev. 99, 1128 (1955).

42. A. I. Akhiezer, V. G. Bar'yakhtar, and S. V. Peletminskii, Spin Waves (North-Holland, Amsterdam, 1968).

43. L. D. Landau and E. M. Lifshitz, Electrodynamics of Continuous Media (Pergamon, Oxford, 1984).

44. A. V. Syromyatnikov, Anomalously large damping of longwavelength quasiparticles caused by long-range interaction, Phys. Rev. B 82, 024432 (2010). 